\title{
Molecular gas in normal late-type galaxies`
}

\author{
A. Boselli ${ }^{1}$, J. Lequeux ${ }^{2}$, and G. Gavazzi ${ }^{3}$ \\ 1 Laboratoire d'Astrophysique de Marseille, BP 8, Traverse du Siphon, 13376 Marseille Cedex 12, France \\ 2 DEMIRM and URA 336 du CNRS, Observatoire de Paris, 61 Av. de l'Observatoire, 75014 Paris, France \\ e-mail: James. Lequeux@obspm.fr \\ 3 Università degli Studi di Milano-Bicocca, Dipartimento di Fisica, Piazza dell'Ateneo Nuovo 1, \\ 20126 Milano, Italy \\ e-mail: Giuseppe.Gavazzi@mib.infn.it
}

Received 7 September 2001 / Accepted 28 November 2001

\begin{abstract}
We present ${ }^{12} \mathrm{CO}(J=1-0)$ line observations of 22 low-luminosity spiral galaxies in the Virgo cluster. These data, together with 244 others available in the literature, allow us to build a large sample that we use to study the molecular gas properties of galaxies spanning a large range of morphological types and luminosities and belonging to different environments (clusters - field). The molecular gas content of the target galaxies is estimated using a luminosity-dependent $X=N\left(\mathrm{H}_{2}\right) / I(\mathrm{CO})$ conversion factor that has been calibrated on a sample of nearby galaxies. $X$ spans from $\sim 10^{20} \mathrm{~mol} \mathrm{~cm}{ }^{-2}\left(\mathrm{~K} \mathrm{~km} \mathrm{~s}^{-1}\right)^{-1}$ in giant spirals to $\sim 10^{21} \mathrm{~mol} \mathrm{~cm}^{-2}\left(\mathrm{~K} \mathrm{~km} \mathrm{~s}^{-1}\right)^{-1}$ in dwarf irregulars. The value of the $X$ conversion factor is found consistent with a value derived independently from dust masses estimated from FIR fluxes, with a metallicity-dependent dust to gas ratio. The relationships between $X$ and the UV radiation field (as traced by the $\mathrm{H} \alpha+[\mathrm{NII}] \mathrm{EW}$ ), the metallicity and the $H$ band luminosity are analysed. We show that the molecular gas contained in molecular clouds or complexes is of the order of $15 \%$ of the total gas on average whatever the luminosity or the Hubble type of the galaxies. We discuss the relation between the star formation rate and the molecular gas content and estimate the average star formation efficiency of late-type galaxies.
\end{abstract}

Key words. galaxies: general, spiral - ISM - galaxies: intergalactic medium - radio lines: galaxies

\section{Introduction}

Our understanding of galaxy evolution is still limited by the poor knowledge of the physical mechanisms behind the process of star formation, i.e. the condensation of the primordial gaseous material (HI) into molecular clouds $\left(\mathrm{H}_{2}\right)$, then their collapse into stars. Also poorly understood are the feed-back processes induced by the newly born stars on the interstellar medium (ISM). The UV radiation field associated with massive star formation produces the ionization of the surrounding gas and causes the photo-dissociation of the hydrogen molecules. Meanwhile supernova winds inject kinetic energy (and metals) into the ISM with the effect of triggering new star formation on the shock fronts.

The first and last stages of this complex chain of transformations are constrained observationally: the atomic hydrogen is directly and easily observable through the $21 \mathrm{~cm}$ line, and the star formation rate (SFR) can be estimated

Send offprint requests to: A. Boselli, e-mail: Alessandro.Boselli@astrsp-mrs.fr

* Based on observations made with the 12-m National Radio Astronomical Observatory, Kitt Peak, Arizona. from $\mathrm{H} \alpha$ and UV luminosities, once corrected for extinction, using stellar population synthesis models (Kennicutt 1998).

Kennicutt (1989) suggested the existence of a universal relationship between gas surface density at disc scales and star formation activity, known as the Schmidt law (Schmidt 1959), modulated by differential rotation. Even if the total amount of gas in late-type galaxies is dominated by atomic hydrogen (Boselli et al. 1997b), which commonly spans a diameter $\sim 1.8$ times the optical disc (Cayatte et al. 1994), the molecular phase might dominate the gas column density inside the optical disc (Young \& Scoville 1991). An accurate determination of the content and distribution of the molecular gas is thus crucial for studying the interplay between gas and star formation over galactic discs.

The amount of molecular hydrogen, can only be estimated by indirect methods. The most common method starts from the ${ }^{12} \mathrm{CO}(1-0)$ line emission at $115 \mathrm{GHz}$ using a standard $X$ conversion factor between the intensity of the $\mathrm{CO}$ line $(I(\mathrm{CO}))$ and the column density of $\mathrm{H}_{2}\left(N\left(\mathrm{H}_{2}\right)\right)$ calibrated in the solar neighborhood, $X=$ $N\left(\mathrm{H}_{2}\right) / I(\mathrm{CO})$ (Young \& Scoville 1991). Detailed analyses 
of giant molecular clouds (GMC) in nearby galaxies have however questioned the validity of this method. $X$ can vary by up to a factor of $\sim 10$ as observed in giant molecular clouds in the SMC (Rubio et al. 1993) or in M 31 (Allen \& Lequeux 1993). Higher values of $X$ are generally observed at low metallicities and strong UV radiation fields.

Using the few local galaxies for which $X$ has been determined with independent methods, it might be possible to calibrate some empirical relationships between $X$ and other parameters characterizing the ISM, such as metallicity, intensity of the UV radiation field and cosmic ray density. This approach is however not easy since the determination of $X$ relies on molecular gas mass estimates based on different techniques: from the virial equilibrium of GMCs (Young \& Scoville 1991), from the line ratios of different CO isotopes (Wild et al. 1992), assuming a metallicity-dependent gas to dust ratio (Guélin et al. 1993, 1995), or relying on $\gamma$-ray data (Hunter et al. 1997). Furthermore the relation between the $\mathrm{CO}$ emission properties and the physical parameters of the ISM (UV radiation field, cosmic ray density, metallicity, gas density) are still poorly known, even though some success in modeling the ISM has recently been reported (Lequeux et al. 1994; Kaufman et al. 1999; Bolatto et al. 1999).

An ideal approach in determining $X$ would be to use a consistent method on a sample of galaxies spanning a large range in luminosity and morphological type. The technique applied by Guélin and collaborators to a few nearby galaxies (Guélin et al. 1993, 1995; Neininger et al. 1996; see also Israel 1997) seems very promising. It consists of determining the amount of dust from millimetric/submillimetric observations and correlating it with $\mathrm{CO}$ and HI. Provided that the gas to dust ratio is constant or metallicity-dependent in a known way, the molecular gas mass can be determined assuming that the difference between the expected total gas to dust ratio and the observed HI to dust ratio is due to molecular gas. This technique could be profitably applied to larger galaxy samples provided that the necessary multifrequency data are available.

For the last few years we have been gathering spectrophotometric data from the UV to the radio centimetric domain for a large sample of $\sim 2500$ galaxies in the nearby Universe. The database created so far is ideal for this purpose since it includes the data necessary for the determination of $X$ (HI, CO and far-IR fluxes) as well as those needed to characterize the physical properties of the ISM ( $\mathrm{H} \alpha$ fluxes as tracers of the UV radiation field and metallicity measurements). The present dataset is however still limited to the bright end of the luminosity function. In particular we are lacking $\mathrm{CO}$ and metallicity measurements for galaxies with $M_{B}>-18$.

In order to extend the available $\mathrm{CO}$ data to lower luminosities, we observed 22 galaxies with $-19<M_{B}<-16$ in the Virgo cluster. The new observations are described in Sects. 2-4. These new data are combined in Sect. 5 with the many data points already available for the galaxies included in our database in order to derive $X$ and then their molecular content. The results of our analysis are discussed and compared with those obtained for a sample of 14 nearby galaxies with independent measurements of $X$ (Sect. 6). The agreement in the determination of $X$ allows us to calibrate a luminosity-dependent $X$ conversion factor which is later used to re-analyse the molecular gas statistical properties of late-type galaxies (Sect. 7). After correcting for the systematic trend of $X$ with luminosity, we estimate the molecular gas fraction and the star formation efficiency of galaxies spanning a large range in luminosity and morphological type and belonging to different environments (Sect. 7).

\section{The new sample}

We report on new observations of the ${ }^{12} \mathrm{CO}(1-0)$ line emission of 22 galaxies belonging to the Virgo Cluster. These are mostly low luminosity late-type galaxies.

The target galaxies are listed in Table 1, arranged as follows:

- Column 1: VCC designation (Binggeli et al. 1985).

- Columns 2 and 3: NGC/IC and UGC names.

- Columns 4 and 5: 1950 celestial coordinates with a few arcsec accuracy, from Binggeli et al. (1985).

- Column 6: morphological type, from Binggeli et al. (1985).

- Column 7: membership of the Virgo cluster, according to Gavazzi et al. (1999b).

- Column 8: heliocentric velocity, in $\mathrm{km} \mathrm{s}^{-1}$, from Binggeli et al. (1985).

- Column 9: distance in Mpc, determined from the cluster membership as described in Gavazzi et al. (1999b).

- Columns 10 and 11: Galaxy major and minor optical blue diameters, in arcmin, from Binggeli et al. (1985). These are isophotal diameters at the faintest observable magnitude.

- Column 12: photographic magnitude as given in the VCC.

- Column 13: width of the HI line (in $\mathrm{km} \mathrm{s}^{-1}$ ) calculated averaging the width at $20 \%$ and at $50 \%$ of the maximum intensity, from Helou et al. (1984), Hoffman et al. (1987, 1989), Schneider et al. (1990), Haynes \& Giovanelli (1986), Magri (1994).

- Column 14: total extrapolated $H$ magnitude, from Boselli et al. (1997a, 2000).

\section{The observations}

The observations were carried out during a remote-observing run (120 hours) from the Laboratoire d'Astrophysique de Marseille in June 2000 using the NRAO Kitt Peak $12 \mathrm{~m}$ telescope ${ }^{1}$. At $115 \mathrm{GHz}\left[{ }^{12} \mathrm{CO}(1-0)\right]$, the telescope

\footnotetext{
1 The Kitt Peak 12-m telescope was operated by Associated Universities, Inc., under cooperative agreement with the National Science Foundation.
} 
Table 1. The target galaxies.

\begin{tabular}{|c|c|c|c|c|c|c|c|c|c|c|c|c|c|}
\hline VCC & $\mathrm{NGC} / \mathrm{IC}$ & UGC & $\begin{array}{c}\mathrm{RA}(1950) \\
\mathrm{h} \mathrm{m} \text { s }\end{array}$ & $\begin{array}{c}\operatorname{dec}(1950) \\
\text { o }, / 1\end{array}$ & type & agg & $\mathrm{km} \mathrm{s}^{-1}$ & $\begin{array}{l}\text { dist } \\
\mathrm{Mpc}\end{array}$ & a & $b$ & $m_{\mathrm{pg}}$ & $\begin{array}{c}\Delta V_{\mathrm{HI}} \\
\mathrm{km} \mathrm{s}^{-1}\end{array}$ & $H_{\mathrm{T}}$ \\
\hline 58 & IC 769 & 7209 & 120959.20 & 122407.0 & $\mathrm{Sb}$ & M & 2207 & 32.0 & 2.54 & 1.75 & 13.17 & 252 & 10.51 \\
\hline 87 & - & - & 121108.20 & 154354.0 & $\mathrm{Sm}$ & $\mathrm{N}$ & -134 & 17.0 & 1.45 & 0.72 & 15.00 & 109 & 13.64 \\
\hline 92 & 4192 & 7231 & 121115.50 & 151042.0 & $\mathrm{Sb}$ & $\mathrm{N}$ & -135 & 17.0 & 9.78 & 2.60 & 10.92 & 469 & 7.07 \\
\hline 97 & 4193 & 7234 & 121120.80 & 132703.0 & $\mathrm{Sc}$ & M & 2476 & 32.0 & 1.96 & 0.97 & 13.20 & 367 & 9.61 \\
\hline 199 & 4224 & 7292 & 121400.50 & 074424.0 & $\mathrm{Sa}$ & W & 2594 & 32.0 & 2.92 & 1.00 & 12.95 & 554 & 8.87 \\
\hline 318 & IC 776 & 7352 & 121630.50 & 090802.0 & Scd & W & 2469 & 32.0 & 1.71 & 1.00 & 14.01 & 178 & 12.95 \\
\hline 459 & - & - & 121839.60 & 175457.0 & BCD & A & 2108 & 17.0 & 0.84 & 0.36 & 14.95 & 127 & 12.70 \\
\hline 792 & 4380 & 7503 & 122249.60 & 101738.0 & Sab & B & 971 & 23.0 & 3.52 & 1.75 & 12.36 & 290 & 8.55 \\
\hline 874 & 4405 & 7529 & 122335.50 & 162728.0 & $\mathrm{Sc}$ & A & 1738 & 17.0 & 1.89 & 1.11 & 12.99 & 169 & 9.61 \\
\hline 939 & - & 7546 & 122414.70 & 090940.0 & $\mathrm{Sc}$ & B & 1271 & 23.0 & 3.45 & 3.45 & 12.92 & 91 & 10.53 \\
\hline 957 & 4420 & 7549 & 122425.20 & 024618.0 & $\mathrm{Sc}$ & $\mathrm{S}$ & 1695 & 17.0 & 2.01 & 0.85 & 12.67 & 214 & 9.87 \\
\hline 1205 & 4470 & 7627 & 122705.30 & 080559.0 & $\mathrm{Sc}$ & $\mathrm{S}$ & 2339 & 17.0 & 1.84 & 1.15 & 13.04 & 147 & 10.22 \\
\hline 1290 & 4480 & 7647 & 122753.40 & 043127.0 & $\mathrm{Sb}$ & $\mathrm{S}$ & 2438 & 17.0 & 2.01 & 1.07 & 13.09 & 319 & 9.80 \\
\hline 1375 & - & 7668 & 122906.20 & 041256.0 & $\mathrm{Sc}$ & S & 1732 & 17.0 & 4.76 & 3.77 & 12.00 & 173 & 11.17 \\
\hline 1412 & 4503 & 7680 & 122934.20 & 112708.0 & $\mathrm{Sa}$ & A & 1342 & 17.0 & 4.33 & 1.71 & 12.12 & - & 8.28 \\
\hline 1508 & 4519 & 7709 & 123057.90 & 085549.0 & $\mathrm{Sc}$ & $\mathrm{S}$ & 1212 & 17.0 & 3.60 & 2.60 & 12.34 & 187 & 9.69 \\
\hline 1554 & 4532 & 7726 & 123146.70 & 064439.0 & $\mathrm{Sm}$ & $\mathrm{S}$ & 2021 & 17.0 & 2.60 & 1.00 & 12.30 & 185 & 9.76 \\
\hline 1686 & IC 3583 & 7784 & 123412.30 & 133202.0 & $\mathrm{Sm}$ & A & 1122 & 17.0 & 2.79 & 1.71 & 13.95 & 116 & 11.19 \\
\hline 1929 & 4633 & 7874 & 124006.50 & 143748.0 & Scd & $\mathrm{E}$ & 291 & 17.0 & 2.48 & 1.07 & 13.77 & 190 & 10.75 \\
\hline 1943 & 4639 & 7884 & 124021.50 & 133152.0 & $\mathrm{Sb}$ & $\mathrm{E}$ & 1048 & 17.0 & 3.20 & 2.01 & 12.19 & 295 & 8.90 \\
\hline 2023 & IC 3742 & 7932 & 124300.90 & 133615.0 & $\mathrm{Sc}$ & $\mathrm{E}$ & 958 & 17.0 & 2.01 & 1.00 & 13.86 & 186 & 11.57 \\
\hline- & 4866 & 8102 & 125657.70 & 142626.0 & $\mathrm{Sa}$ & $\mathrm{E}$ & 1986 & 17.0 & 6.00 & 1.44 & 11.90 & 537 & 8.19 \\
\hline
\end{tabular}

half-power beam width (HPBW) is $55^{\prime \prime}$ which corresponds to $4.5 \mathrm{kpc}$ at the assumed distance of $17 \mathrm{Mpc}$ for the Virgo cluster. Weather conditions were fairly good, with typical zenith opacities of $0.30-0.45$. The pointing accuracy was checked every night by broad band continuum observations of Saturn and/or 3C 273, with an average error of $7^{\prime \prime}$ rms. We used a dual-polarization SIS mixer, with a receiver temperature for each polarisation of about $T_{\text {sys }}=350-600 \mathrm{~K}$ (in $T_{\mathrm{R}}^{*}$ scale) at the elevation of the sources. We used a dual beam-switching procedure, with two symmetric reference positions offset by $4^{\prime}$ in azimuth. The backend was a 256 channel filter bank spectrometer with channel width of $2 \mathrm{MHz}$. Each 6-min scan began by a chopper wheel calibration on a load at ambient temperature, with a chopper wheel calibration on a cold load every two scans. Galaxies were observed at their nominal coordinates listed in Table 1, with one position per galaxy. The total integration time was on average $120 \mathrm{~min}$ on+off (i.e. $60 \mathrm{~min}$ on the source), yielding rms noise levels of about $3 \mathrm{mK}$ (in the $T_{\mathrm{R}}^{*}$ scale) after velocity smoothing to $21 \mathrm{~km} \mathrm{~s}^{-1}$. The baselines were flat owing to the use of beam-switching, thereby requiring that only linear baselines be subtracted. The antenna temperature $T_{\mathrm{R}}^{*}$ was corrected for telescope and atmospheric losses. In the following analysis we use the main-beam brightness temperature scale, $T_{\mathrm{mb}}$, with $T_{\mathrm{mb}}=T_{\mathrm{R}}^{*} / 0.84$ (where the main beam efficiency is $\eta_{\mathrm{mb}}=0.54$ and the forward scattering and spillover efficiency $\eta_{\text {fss }}=0.68$ ). This scale is appropriate for sources with sizes comparable to, or smaller than, the beam size. These main-beam temperatures can be converted into flux densities using $29 \mathrm{Jy} / \mathrm{K}$.

\section{Results}

\subsection{Results of our observations}

The ${ }^{12} \mathrm{CO}(1-0)$ spectra of all the detected galaxies, reduced with the CLASS package (Forveille et al. 1990), are shown in Fig. 1: the observational results are listed in Table 2 . Of the 22 observed galaxies 11 were not detected. Table 2 is arranged as follows:

- Column 1: VCC name.

- Column 2: integration time (on+off), in minutes.

- Column 3: rms noise, in $\mathrm{mK}$, in the $T_{\mathrm{R}}^{*}$ scale.

- Column 4: intensity of the $I(\mathrm{CO})$ line $(I(\mathrm{CO})=$ $\left.\int T_{\mathrm{R}}^{*} \mathrm{~d} v\right)$ in $\mathrm{K} \mathrm{km} \mathrm{s}^{-1}$ (area definition)). For undetected galaxies, the reported value is an upper limit determined as follows:

$I(\mathrm{CO})=2 \sigma\left(\Delta V_{\mathrm{HI}} \delta V_{\mathrm{CO}}\right)^{1 / 2} \mathrm{~K} \mathrm{~km} \mathrm{~s}^{-1}$

where $\sigma$ is the rms noise of the spectrum, $\Delta V_{\mathrm{HI}}$ is the HI line width, and $\delta V_{\mathrm{CO}}$ is the spectral resolution (for galaxies with $\Delta V_{\mathrm{HI}}$ not available, the $\mathrm{HI}$ width has been determined assuming a standard $\Delta V_{\mathrm{HI}}=$ $300 \sin (i) \mathrm{km} \mathrm{s}^{-1}$, where $i$ is the galaxy inclination or $\Delta V_{\mathrm{HI}}=50 \mathrm{~km} \mathrm{~s}^{-1}$ if $\left.i=0\right) . \delta V_{\mathrm{CO}}=21 \mathrm{~km} \mathrm{~s}^{-1}$.

- Column 5: error on the intensity of the CO line, $\Delta I(\mathrm{CO})$, computed as:

$\Delta I(\mathrm{CO})=2 \sigma\left(\Delta V_{\mathrm{CO}} \delta V_{\mathrm{CO}}\right)^{1 / 2} \mathrm{~K} \mathrm{~km} \mathrm{~s}^{-1}$

where $\sigma$ is the rms noise of the spectrum, $\Delta V_{\mathrm{CO}}$ is the $\mathrm{CO}$ linewidth (given in Col. 7 ), and $\delta V_{\mathrm{CO}}$ is the 

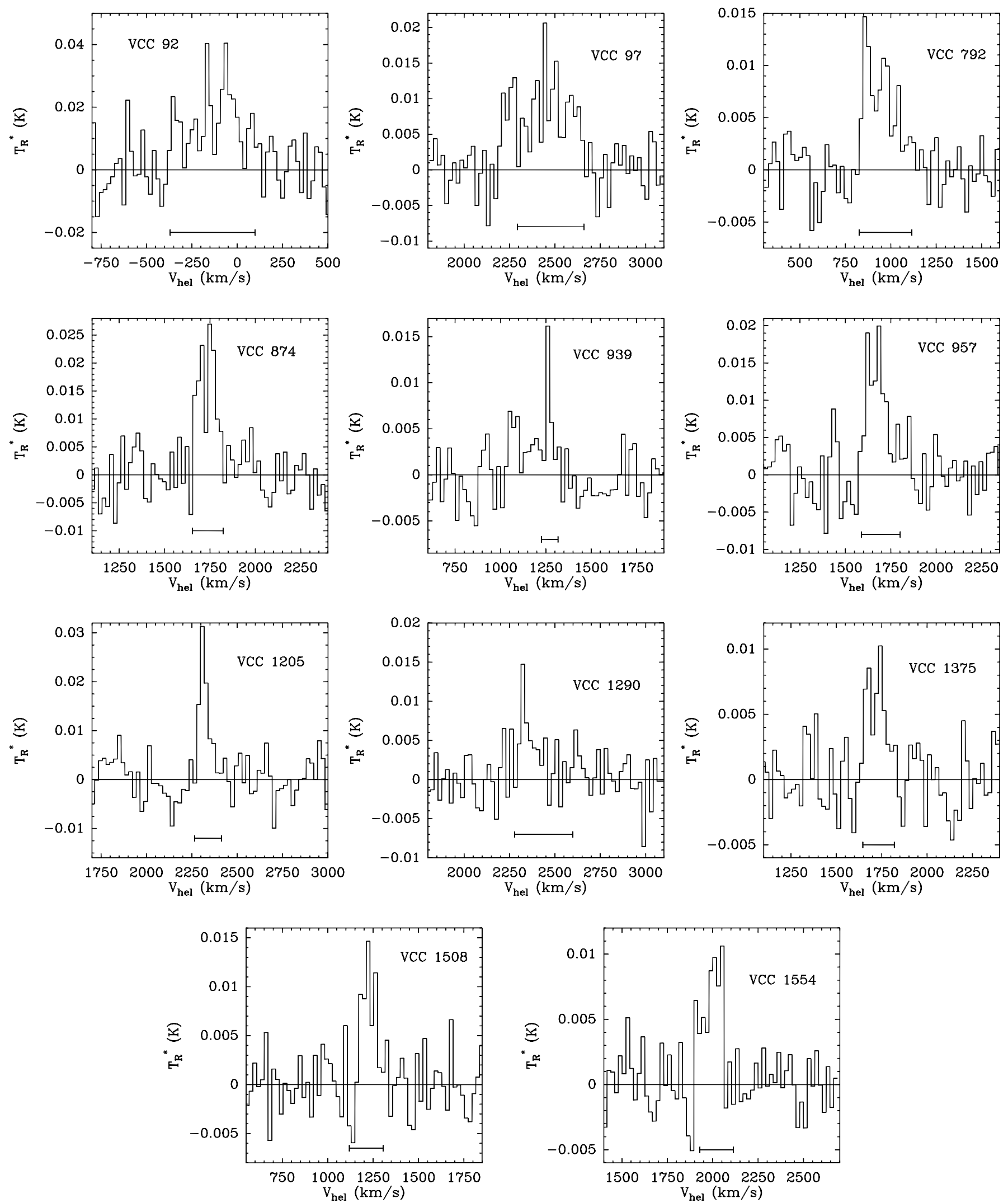

Fig. 1. ${ }^{12} \mathrm{CO}(1-0)$ line spectra (smoothed to $\delta V_{\mathrm{CO}}=21 \mathrm{~km} \mathrm{~s}^{-1}$ ) of the 11 detected galaxies (in order of increasing VCC name) in the $T_{\mathrm{R}}^{*}$ scale. The horizontal line indicates the HI line width listed in Table 1.

spectral resolution. Spectra were smoothed to $\delta V_{\mathrm{CO}}=$ $21 \mathrm{~km} \mathrm{~s}^{-1}$.
- Column 6: heliocentric velocity determined from the $\mathrm{CO}$ line (Gaussian fit), in $\mathrm{km} \mathrm{s}^{-1}$ (optical definition 
$\left.v=c z=\Delta \lambda / \lambda_{0}\right)$. The estimated error is comparable to the resolution, thus $\sim 20 \mathrm{~km} \mathrm{~s}^{-1}$.

- Column \%: width at $50 \%$ of the maximum intensity of the $\mathrm{CO}$ line, in $\mathrm{km} \mathrm{s}^{-1}$, with an estimated error of $\sim 20 \mathrm{~km} \mathrm{~s}^{-1}$.

- Column 8: the $M\left(\mathrm{H}_{2}\right)_{\mathrm{CO}}$ mass, determined as in Sect. 4.2 adopting a standard $X$ conversion factor $X=2.3 \times 10^{20} \mathrm{~mol} \mathrm{~cm}^{-2}\left(\mathrm{~K} \mathrm{~km} \mathrm{~s}^{-1}\right)^{-1}$.

- Column 9: filling factor, defined as the ratio of the beam area to the optical surface of the galaxy.

- Column 10: the molecular gas mass determined from the CO line intensity as described in Sect. 7 assuming the $H$ luminosity-dependent $X$ conversion factor given in Table 5.

\subsection{Comparison with previous observations}

$\mathrm{CO}$ measurements are often used to estimate the molecular hydrogen content of galaxies adopting a standard conversion factor from $\mathrm{CO}$ intensities to $\mathrm{H}_{2}$ column densities $(X)$. In order to allow a comparison with previous works we convert the $\mathrm{CO}$ line fluxes into $\mathrm{H}_{2}$ masses using the conventional Galactic conversion factor $X=2.3 \times$ $10^{20} \mathrm{~mol} \mathrm{~cm}^{-2}\left(\mathrm{~K} \mathrm{~km} \mathrm{~s}^{-1}\right)^{-1}$ of Strong et al. (1988) where $I(\mathrm{CO})$ from Col. 4 of Table 2 is converted into the $T_{\mathrm{mb}}$ scale (see Sect. 3). The molecular gas mass $\left(M\left(\mathrm{H}_{2}\right)_{\mathrm{CO}}\right.$, in solar units) as determined from $\mathrm{CO}$ measurements, is given by:

$M\left(\mathrm{H}_{2}\right)_{\mathrm{CO}}=68.08 \times(D / \mathrm{Mpc})^{2} I(\mathrm{CO})\left(\mathrm{K} \mathrm{km} \mathrm{s}^{-1}\right)\left(\theta / 1^{\prime \prime}\right)^{2}(3)$

where $\theta$ is the half-power beam width (HPBW) of the telescope and $D$ the distance to the source (as given in Table 1).

Since the galaxies were observed only at the central position, our mass determinations should be considered as lower limits of the total $M\left(\mathrm{H}_{2}\right)_{\mathrm{CO}}$. However since the optical angular sizes of the observed galaxies do not generally exceed the adopted beam by more than a factor of two, and since the $\mathrm{CO}$ emission in spiral galaxies is centrally peaked, with an exponential distribution with a scale length $\approx 1.5$ smaller than the optical one (Young et al. 1995), the present data should give reliable estimates of the total CO emission.

Seven target galaxies were previously observed using the $13.7 \mathrm{~m}$ FCRAO radiotelescope, which, at this frequency, has a beam size of 45 arcsec, thus comparable to the NRAO $12 \mathrm{~m}$. We compare in Table 3 the different sets of data. The intensities given in Table 3 are transformed to the main beam scale adopting a main beam efficiency $\eta_{\mathrm{mb}}=0.53$ for the FCRAO. Masses are estimated adopting the same $\mathrm{CO}$ to $\mathrm{H}_{2}$ standard conversion factor $X=2.3 \times 10^{20} \mathrm{~mol} \mathrm{~cm}{ }^{-2}\left(\mathrm{~K} \mathrm{~km} \mathrm{~s}^{-1}\right)^{-1}$ and distance (as given in Table 1). For galaxies observed at several positions, we compare the central beam observations.

In spite of the different beam sizes of the telescopes, the two sets of CO intensity determinations are consistent within a factor of 2 . Our observations are generally more sensitive than the published ones, except for VCC 92
(NGC 4192), which was observed as a cross check, and VCC 1943 (NGC 4639), which was interrupted because of incoming bad weather.

\section{Data analysis}

\subsection{The samples}

The molecular gas properties of late-type galaxies are analysed in this section using the presently reported data jointly with those available in the literature for a large sample of optically selected galaxies in the Coma/A1367 supercluster and in the Virgo cluster (Boselli et al. 1997b, 1995a and references therein) extracted respectively from the Zwicky catalogue (CGCG) and from the Virgo cluster catalogue of Binggeli et al. (1985, VCC). The sample, which is not complete in any sense, includes 266 normal galaxies with ${ }^{12} \mathrm{CO}(1-0)$ data spanning a large range in morphological type (Sa to Im and BCD) and luminosity $\left(-16>M_{B}>-22\right)$.

The accuracy of the morphological classification is excellent for the Virgo galaxies (Binggeli et al. 1985, 1993). Because of the higher distance, the morphology of galaxies belonging to the other surveyed regions suffers from an uncertainty of about \pm 0.75 Hubble type bins.

We assume a distance of $17 \mathrm{Mpc}$ for the members (and possible members) of Virgo cluster A, $22 \mathrm{Mpc}$ for Virgo cluster B, $32 \mathrm{Mpc}$ for objects in the $\mathrm{M}$ and $\mathrm{W}$ clouds (see Gavazzi et al. 1999b). Members of the Coma and A1367 clusters are assumed to be at distances of 86.6 and $92 \mathrm{Mpc}$ respectively. Isolated galaxies in the Coma supercluster are assumed to be at their redshift distance adopting $H_{0}=75 \mathrm{~km} \mathrm{~s}^{-1} \mathrm{Mpc}^{-1}$.

Three different subsamples can be extracted from the Coma/A1367 supercluster and Virgo cluster galaxies:

i) the unperturbed sample is composed of 153 latetype galaxies, both isolated or cluster members, whose HIdeficiency (defined as the logarithm of the ratio of the HI mass to the average HI mass of isolated objects of similar morphological type and linear size, Haynes \& Giovanelli 1984) is HI - def $\leq 0.3$.

ii) the isolated sample is composed of 47 strictly isolated galaxies in the bridge between Coma and A1367 (see Gavazzi et al. 1999a).

iii) the ISOPHOT sample consists of 18 galaxies in Coma and A1367 observed by ISOPHOT (see next section; Contursi et al. 2001).

A small sample of 14 nearby galaxies with an independent measure of the $X$ conversion factor is described in Table 4.

\subsection{The complementary data}

Multifrequency spectrophotometric data are available for most of the analysed galaxies.

The CO data (266 sources) are taken from Boselli et al. (1995a, 1997b), Kenney \& Young (1988a), Young et al. (1995) and references therein. For mapped galaxies (most 
Table 2. Results of the observations, in the $T_{\mathrm{R}}^{*}$ scale.

\begin{tabular}{rrrrrrrrrr}
\hline name & $\begin{array}{r}\text { int - time } \\
\text { min(on + off })\end{array}$ & $\begin{array}{r}\mathrm{rms} \\
\mathrm{mK}\end{array}$ & $\begin{array}{r}I(\mathrm{CO}) \\
\mathrm{K} \mathrm{km} \mathrm{s}^{-1}\end{array}$ & $\begin{array}{c}\Delta I(\mathrm{CO}) \\
\mathrm{K} \mathrm{km} \mathrm{s}^{-1}\end{array}$ & $\begin{array}{r}V(\mathrm{CO}) \\
\mathrm{km} \mathrm{s}^{-1}\end{array}$ & $\begin{array}{r}\Delta V_{\mathrm{CO}} \\
\mathrm{km} \mathrm{s}^{-1}\end{array}$ & $\begin{array}{r}\log M\left(\mathrm{H}_{2}\right)_{\mathrm{CO}} \\
M_{\odot}\end{array}$ & $\begin{array}{r}F F \\
\log M\left(\mathrm{H}_{2}\right) \\
M_{\odot}\end{array}$ \\
\hline 58 & 162 & 3 & $<0.44$ & - & - & - & $<8.04$ & 0.19 & $<8.10$ \\
87 & 156 & 4 & $<0.38$ & - & - & - & $<7.43$ & 0.80 & $<8.15$ \\
92 & 30 & 9 & 7.66 & 1.56 & -102 & 360 & 8.74 & 0.04 & 8.49 \\
97 & 96 & 3 & 3.89 & 0.55 & 2442 & 400 & 8.99 & 0.44 & 8.91 \\
199 & 240 & 3 & $<0.86$ & - & - & - & $<8.21$ & 0.29 & $<8.01$ \\
318 & 132 & 3 & $<0.37$ & - & - & - & $<7.96$ & 0.49 & $<8.37$ \\
459 & 204 & 2 & $<0.21$ & - & - & - & $<7.17$ & 2.78 & $<7.75$ \\
792 & 192 & 2 & 1.86 & 0.27 & 934 & 210 & 8.38 & 0.14 & 8.25 \\
874 & 120 & 4 & 2.53 & 0.40 & 1736 & 120 & 8.25 & 0.40 & 8.37 \\
939 & 240 & 3 & 0.52 & 0.13 & 1265 & 20 & 7.83 & 0.07 & 8.00 \\
957 & 174 & 4 & 2.30 & 0.37 & 1669 & 100 & 8.21 & 0.49 & 8.36 \\
1205 & 120 & 4 & 1.72 & 0.23 & 2312 & 40 & 8.09 & 0.40 & 8.30 \\
1290 & 198 & 3 & 1.23 & 0.17 & $2336 *$ & 40 & 7.94 & 0.39 & 8.09 \\
1375 & 270 & 2 & 0.93 & 0.21 & 1720 & 130 & 7.81 & 0.05 & 8.17 \\
1412 & 114 & 7 & $<1.07$ & - & - & - & $<7.88$ & 0.11 & $<7.81$ \\
1508 & 408 & 3 & 1.08 & 0.29 & 1226 & 110 & 7.88 & 0.09 & 8.02 \\
1554 & 222 & 2 & 1.17 & 0.24 & 2006 & 170 & 7.92 & 0.32 & 8.08 \\
1686 & 168 & 4 & $<0.39$ & - & - & - & $<7.45$ & 0.18 & $<7.81$ \\
1929 & 228 & 3 & $<0.38$ & - & - & - & $<7.43$ & 0.32 & $<7.72$ \\
1943 & 78 & 10 & $<1.57$ & - & - & - & $<8.05$ & 0.13 & $<7.70$ \\
2023 & 282 & 4 & $<0.50$ & - & - & - & $<7.55$ & 0.42 & $<7.96$ \\
$\mathrm{~N} 4866$ & 168 & 4 & $<0.85$ & - & - & - & $<7.78$ & 0.10 & $<7.67$ \\
\hline
\end{tabular}

* Poor quality fit.

Table 3. Comparison between our data $(\mathrm{TW})$ and those available in the literature (L).

\begin{tabular}{rrrrrrr}
\hline name & $\begin{array}{c}\mathrm{rms} \\
\mathrm{mK}\end{array}$ & $\begin{array}{r}I(\mathrm{CO}) \\
\mathrm{K} \mathrm{km} \mathrm{s}^{-1}\end{array}$ & $\begin{array}{c}\delta V_{\mathrm{CO}} \\
\mathrm{km} \mathrm{s}^{-1}\end{array}$ & $\begin{array}{r}\log M\left(\mathrm{H}_{2}\right)_{\mathrm{CO}} \\
M_{\odot}\end{array}$ & $\begin{array}{r}\log M\left(\mathrm{H}_{2}\right)_{\mathrm{CO}_{\mathrm{L}}} \\
M_{\odot}\end{array}$ & reference \\
\hline 92 & 13 & 9.29 & 12 & 8.74 & 8.57 & $\mathrm{KY}$ \\
792 & 15 & $<1.65$ & 12 & 8.38 & $<8.08$ & $\mathrm{KY}$ \\
1412 & 20 & $<2.63$ & 15 & $<7.88$ & $<8.02$ & $\mathrm{Y}$ \\
1508 & 20 & $<1.90$ & 12 & 7.88 & $<7.88$ & $\mathrm{~K}$ \\
1554 & 14 & $<1.32$ & 12 & $<.92$ & $<7.72$ & $\mathrm{KY}$ \\
1943 & 10 & $<1.20$ & 12 & $<8.05$ & $<7.89$ & $\mathrm{KY}$ \\
$\mathrm{N} 4866$ & 12 & $<1.95$ & 12 & $<7.78$ & $<$ & $\mathrm{KY}$ \\
\hline
\end{tabular}

KY: Kenney \& Young (1988a); Y: Young et al. (1995); K: Kenney, private communication.

of the bright Virgo spirals), the total CO emission is estimated as the sum of the emission in each single pointing. The error on the CO line intensity is approximately $\pm 10 \%$.

211 of the sample galaxies have been observed in $\mathrm{H} \alpha$. $\mathrm{H} \alpha+[\mathrm{NII}]$ fluxes obtained from imaging, aperture photometry or integrated spectra are taken from Kennicutt \& Kent (1983), Kennicutt et al. (1984), Gavazzi et al. (1991) Gavazzi et al. (1998), Moss et al. (1998), and references therein. Additional observations of several galaxies have been recently obtained by us during several runs at the Observatoire de Haute Provence (France), at San Pedro Martir (Mexico) and at Calar Alto (Boselli \& Gavazzi 2002; Gavazzi et al. 2002). $\mathrm{H} \alpha+[\mathrm{NII}] \mathrm{EW}$ (equivalent widths) from Kennicutt \& Kent (1983), have been multiplied by 1.16, as suggested by Kennicutt et al. (1994), in order to account for the continuum flux overestimate due to inclusion of the telluric absorption band near $6900 \AA$ in the comparison filter. The estimated error on the $\mathrm{H} \alpha+[\mathrm{NII}] \mathrm{EW}$ is $\sim \pm 7 \%$.

HI fluxes are available for 263 galaxies. HI data are taken from Scodeggio \& Gavazzi (1993) and Hoffman et al. (1996) and references therein. HI fluxes are transformed into neutral hydrogen masses with an uncertainty of $\sim \pm 5 \%$.

NIR data for 255 galaxies, mostly from Nicmos3 observations, are taken from Gavazzi et al. (1996a,b, 2000a), Boselli et al. (1997a, 2000). From these data we derive total (extrapolated to infinity) magnitudes $H_{\mathrm{T}}$, as described in Gavazzi et al. (2000b) with typical uncertainties of $\sim \pm 5 \%$. These are converted into total luminosities using: $\log L_{H}=11.36-0.4 H_{\mathrm{T}}+2 \log D$ (in solar units), where $D$ is the distance to the source (in Mpc). For a few 
objects we derive the $H$ luminosity from $K^{\prime}$ band measurements assuming an average $H-K^{\prime}$ colour of 0.25 mag (independent of type; see Gavazzi et al. 2000a). A minority of the objects in our sample have an $H$ band magnitude obtained from aperture photometry, thus with no asymptotic extrapolation. For these we use the magnitude $H_{25}$ determined as in Gavazzi \& Boselli (1996) at the optical radius (the radius at which the $B$ surface brightness is $25 \mathrm{mag} \operatorname{arcsec}^{-2}$ ) which is on average $0.1 \mathrm{mag}$ fainter than $H_{\mathrm{T}}$ (Gavazzi et al. 2000a,b). The total $H$ magnitudes are corrected for internal extinction according to Gavazzi \& Boselli (1996). No such correction has been applied to galaxies of type $>$ Scd.

Metallicity measurements $12+\log (\mathrm{O} / \mathrm{H})$ are available for a small fraction of the sample (46 galaxies). These are either determined at $r=0.4 \rho_{0}$ for galaxies from individual HII region measurements (Zaritsky et al. 1994), or average estimates if obtained from integrated spectra (Kennicutt 1992; Gavazzi et al. in preparation).

Far-IR fluxes at $100 \mu \mathrm{m}$ from IRAS for 262 galaxies are taken from several compilations such as Bicay \& Giovanelli (1987), Thuan \& Sauvage (1992) and references therein. For 18 galaxies ISOPHOT data in the wavelength range 100-200 $\mu \mathrm{m}$ are also available (Contursi et al. 2001).

The corresponding multifrequency spectrophotometric data for the reference sample of nearby galaxies are listed in Table 4. Giant molecular complexes of angular dimensions larger than 100 pc might be in non-equilibrium. Furthermore the contribution of the atomic hydrogen inside these complexes to their total mass can be important. An accurate estimate of the molecular gas mass using the virial theorem can be obtained only for the dense cores in virial equilibrium of giant molecular clouds, where the contribution of $\mathrm{HI}$ is negligible. These cores have linear dimensions of $\sim 10$ pc. We thus decided to include in Table 4 only those galaxies with interferometric CO observations where the high resolution $(\leq 100 \mathrm{pc})$ allowed the determination of $X$ from CO line widths and intensities measurements of the resolved cores of molecular clouds.

\section{An empirical calibration of}

\subsection{The nearby sample of resolved galaxies}

Table 4 contains the best-estimated values of $X$ for a sample of 14 well-studied nearby galaxies. Table 4 shows that $X$ spans the range $0.6 \leq X \leq 10 \times$ $10^{20} \mathrm{~mol} \mathrm{~cm}^{-2}\left(\mathrm{~K} \mathrm{~km} \mathrm{~s}^{-1}\right)^{-1}$ for galaxies of different morphological type.

The ratio between ${ }^{12} \mathrm{CO}(1-0)$ line emission and the molecular hydrogen column density strongly depends on several physical properties of the ISM such as the UV radiation field, the metallicity and the cosmic ray density (Lequeux et al. 1994; Kaufman et al. 1999), which are known to vary from galaxy to galaxy.

We empirically quantify these dependences by plotting in Fig. 2. (left panels) the relationship between $X$, the
$\mathrm{H} \alpha+[\mathrm{NII}] \mathrm{EW}$ and the metallicity $(12+\log (\mathrm{O} / \mathrm{H}))$ for the 14 nearby galaxies.

The relationship with the $\mathrm{H} \alpha+[\mathrm{NII}] \mathrm{EW}$ (taken as a SFR tracer), if any, is ill-defined and that with metallicity is quite clear. In galaxies with a low metallicity and a strong UV radiation field (high $\mathrm{H} \alpha+[\mathrm{NII}] \mathrm{EW}$ ) (both go together in general) the $X$ conversion factor is a factor of $\sim 20$ higher than in quiescent, high metallicity galaxies such as the Milky Way. These two relationships can be used in principle to determine a more accurate value of the $X$ conversion factor once the metallicity and/or the $\mathrm{H} \alpha+$ $[\mathrm{NII}] \mathrm{EW}$ is known. Metallicity measurements are available for only a minority of galaxies, while $\mathrm{H} \alpha+[\mathrm{NII}] \mathrm{EW}$ exist for a few hundred. However there is a well-known anticorrelation (correlation) between $\mathrm{H} \alpha+[\mathrm{NII}] \mathrm{EW}$ (metallicity) and luminosity (Gavazzi et al. 1998; Zaritsky et al. 1994) in normal galaxies. It is reflected here by a strong relation between $X$ and the $H$ luminosity (Fig. 2c) or the $B$ absolute magnitude (Fig. 2d). The best fits to the data are given in Table 5. The slope of the fits are consistent with those found by Arimoto et al. (1996), but significantely steeper than that found by Wilson (1995) for the $X$ vs. $12+\log (\mathrm{O} / \mathrm{H})$ relation (see Table 5$)$. This difference in slope with Wilson is probably due to the fact that our sample includes many metal rich spiral galaxies with low values of $X\left(X \leq 10^{20} \mathrm{~mol} \mathrm{~cm}^{-2}\left(\mathrm{~K} \mathrm{~km} \mathrm{~s}^{-1}\right)^{-1}\right)$ not present in the Wilson's sample. Our intercept for the $X$ vs. $12+\log (\mathrm{O} / \mathrm{H})$ relation is consistent with that of Arimoto et al. (1996). Our intercept in the $X$ vs. $M_{B}$ relation is lower since Arimoto et al. (1996) includes all the objects rejected here whose $X$ value is probably overestimated due to the low spatial resolution of the $\mathrm{CO}$ observations $(>100 \mathrm{pc})$.

The relationships given in Table 5 between $X$ and $L_{H}$ and/or $M_{B}$ are de facto empirical calibrations for a luminosity-dependent $X$ conversion factor. Given the large uncertainty in the determination of $X$ in the nearby sample of galaxies, it is difficult to quantify the resulting accuracy in the molecular gas mass estimated using the relationships given in Table 5 . We should also remind that even inside a given object $X$ might change by a factor of $\sim 10$ from the diffuse medium to the core of GMCs (Polk et al. 1988); it is thus difficult to estimate a single value of $X$ representing the entire galaxy. We can however conclude that the adoption of the relations given in Table 5 should remove the first-order systematic effect with luminosity in the estimate of the molecular hydrogen content of galaxies using $\mathrm{CO}$ data. The use of a standard $X$ conversion factor as those generally used in the literature $(X=$ $\left.2.3-2.8 \times 10^{20} \mathrm{~mol} \mathrm{~cm}^{-2}\left(\mathrm{~K} \mathrm{~km} \mathrm{~s}^{-1}\right)^{-1}\right)$ overestimates the molecular gas mass by a factor of $\sim 2-3$ in massive galaxies of $L_{H} \sim 10^{11} L_{H \odot}$, or $M_{B} \sim-20.5 \mathrm{mag}$, while underestimates $M\left(\mathrm{H}_{2}\right)$ in low mass objects of $L_{H} \sim 10^{9} L_{H \odot}$, or $M_{B} \sim-17 \mathrm{mag}$ as those observed in this work by a factor of $\sim 2$. The relationship between $X$ and $12+\log (\mathrm{O} / \mathrm{H})$ might be used to estimate the radial distribution of molecular hydrogen in galaxies mapped in $\mathrm{CO}$ with available measurements of the metallicity gradient. 
Table 4. Multifrequency data for the sample of nearby galaxies.

\begin{tabular}{|c|c|c|c|c|c|c|c|c|c|c|c|}
\hline name & type & $\begin{array}{l}\text { dist } \\
\text { Mpc }\end{array}$ & $\begin{array}{l}M_{B}^{a} \\
\text { mag }\end{array}$ & $\begin{array}{l}\text { est. } \log L_{H}^{b} \\
\text { solar units }\end{array}$ & $12+\log (\mathrm{O} / \mathrm{H})$ & ref. & $\begin{array}{c}\mathrm{H} \alpha+[\mathrm{NII}] \mathrm{EW} \\
\AA\end{array}$ & ref. & $\begin{array}{c}X \\
10^{20}\end{array}$ & $\operatorname{method}^{e}$ & ref. \\
\hline MW & & 0 & -20.40 & $10.76^{c}$ & $8.90 \pm 0.04$ & 1 & - & - & $1.56 \pm 0.05$ & gamma & 15 \\
\hline $\mathrm{SMC}$ & $\mathrm{SB}(\mathrm{s}) \mathrm{m}$ & 0.061 & -16.30 & $8.81^{c}$ & $8.04 \pm 0.06$ & 1 & $24 \pm 5$ & 5 & 10.0 & virial & 16 \\
\hline LMC & $\mathrm{SB}(\mathrm{s}) \mathrm{m}$ & 0.055 & -18.40 & $9.81^{c}$ & $8.37 \pm 0.12$ & 1 & $36 \pm 8$ & 5 & 8.0 & virial & 17 \\
\hline M31 & $\mathrm{SA}(\mathrm{s}) \mathrm{b}$ & 0.77 & -20.67 & 11.07 & $9.01 \pm 0.10$ & 1 & $3.7 \pm 1.4^{d}$ & 6 & $2.4 \pm 1.5$ & virial & 1 \\
\hline M33 & $\mathrm{SA}(\mathrm{s}) \mathrm{cd}$ & 0.84 & -18.31 & 9.90 & $8.78 \pm 0.05$ & 2 & $20 \pm 2$ & 7 & $5.0 \pm 1.5$ & virial & 1 \\
\hline M51 & $\mathrm{SA}(\mathrm{s}) \mathrm{bc}$ & 9.6 & -20.75 & 11.08 & $9.23 \pm 0.12$ & 2 & $19 \pm 2.5$ & 8 & 0.6 & $\mathrm{~mm}$ & 16 \\
\hline M81 & $\mathrm{SA}(\mathrm{s}) \mathrm{ab}$ & 3.8 & -20.51 & 10.90 & $9.00 \pm 0.13$ & 2 & $10 \pm 4.5$ & 8 & $0.7^{f}$ & virial & 18,19 \\
\hline M82 & I0sbrst & 3.63 & -18.94 & 10.69 & $9.00 \pm 0.12$ & 3 & $42 \pm 1$ & 8 & 1 & $\mathrm{~mm}$ & 20 \\
\hline IC10 & $\mathrm{IBm}$ & 0.66 & -15.90 & $8.62^{c}$ & $8.31 \pm 0.20$ & 1 & - & - & $6.6 \pm 2.2$ & virial & 1 \\
\hline N891 & $\mathrm{SA}(\mathrm{s}) \mathrm{b} ? \mathrm{Sp}$ & 9.5 & -20.52 & 10.78 & - & - & $7.5 \pm 1$ & $10,11,12,13$ & 1.5 & $\mathrm{~mm}$ & 16,22 \\
\hline N1569 & $\mathrm{IBm}$ & 2.2 & -16.90 & 8.79 & $8.19 \pm 0.02$ & 4 & $149 \pm 15$ & 9,14 & $15^{f}$ & virial & 10 \\
\hline N4565 & $\mathrm{SA}(\mathrm{s}) \mathrm{b} ? \mathrm{Sp}$ & 9.4 & -21.77 & 11.13 & - & - & $2.7 \pm 1.5^{d}$ & 12 & 1.0 & $\mathrm{~mm}$ & 16 \\
\hline N6822 & $\mathrm{IB}(\mathrm{s}) \mathrm{m}$ & 0.5 & -15.10 & 8.15 & $8.16 \pm 0.06$ & 1 & - & - & $6.6 \pm 3.9$ & virial & 1 \\
\hline N6946 & $\mathrm{SAB}(\mathrm{rs}) \mathrm{cd}$ & 5.5 & -20.92 & 10.60 & $9.06 \pm 0.17$ & 2 & $29 \pm 5$ & 14 & 1.8 & $\mathrm{~mm}$ & 21 \\
\hline
\end{tabular}

${ }^{a} B$ total corrected magnitudes are from NED, and distances from van den Bergh (1999); for the MW, $M_{B}$ is from van den Bergh (1999).

${ }^{b} H$ band magnitudes, corrected for extinction as in Gavazzi \& Boselli (1996), are extracted from the 2MASS survey and/or from aperture photometry (Gezari et al. 1993), or form recent observations taken at the 3.5 TNG telescope (Gavazzi et al., in preparation). The estimated error on the $H$ luminosity is $< \pm 10 \%$ (photometric accuracy plus the extrapolation to a total value), $\pm 0.12 \mathrm{mag}$ (in $\log$ ) when determined from the $L_{H}$ vs. $M_{B}$ relation given in c), and \pm 0.15 mag for the Milky Way (MW). ${ }^{c}$ For galaxies not observed in the $H$ band (5), the $H$ luminosity is determined from $\log L_{H}\left(L_{H \odot}\right)=1.05( \pm 0.22)-0.48$ $( \pm 0.01) \times M_{B}\left(R^{2}=0.84\right.$, where $R$ is the regression coefficient) has been determined from 246 galaxies in our sample.

${ }^{d}$ For M 31 and NGC $4565 \mathrm{H} \alpha+[\mathrm{NII}] \mathrm{EW}$ have been estimated using published $\mathrm{H} \alpha$ fluxes and red continuum as determined from the $R$ band photometry assuming $0.0 \mathrm{mag}$ in $R$ equivalent to $1.74 \times 10^{-9} \mathrm{erg} \mathrm{cm}^{-2} \mathrm{~s}^{-1}$. Given its high inclination, we corrected the $\mathrm{H} \alpha$ flux of NGC 4565 for 2 mag.

e Gamma: from high-energy gamma-ray emission from EGRET observations; virial: from CO observations of resolved molecular clouds assuming virial equilibrium; mm: from millimetric observations assuming a metallicity-dependent dust to gas ratio.

${ }^{f}$ Uncertain values

References:

1: Arimoto et al. (1996), average of the disc; 2: Zaritsky et al. (1994); 3: Gavazzi et al., in preparation; 4: Kobulnicky \& Skillman (1997); 5: Kennicutt et al. (1995); 6: H $\alpha$ flux from Devereux et al. (1994), and $R$ magnitude from Walterbos \& Kennicutt (1987); 7: Devereux et al. (1997), and private communication; 8: Boselli \& Gavazzi (2002); 9: Hunter et al. (1993); 10: Taylor et al. (1999); 11: Hoopes, private communication; 12: Rand et al. (1992); 13: Hoopes et al. (1999); 14: Kennicutt \& Kent (1983); 15: Hunter et al. (1997), average over the whole Milky Way; 16: Boselli et al. (1997b), scale of 10 pc for SMC; 17: Rubio (1999), scale 10 pc; 18: Taylor \& Wilson (1998); 19: Allen et al. (1997); 20: Wild et al. (1992), central 1 kpc; 21: Bianchi et al. (2000); 22: Alton et al. (2000).

Table 5. The relationships between the $X$ conversion factor and different galaxy properties. $X$ is defined as: $\log X=$ slope $\times$ Variable + constant (in units of $\left.\mathrm{mol} \mathrm{cm} \mathrm{cm}^{-2}\left(\mathrm{~K} \mathrm{~km} \mathrm{~s}^{-1}\right)^{-1}\right)$.

\begin{tabular}{lcccc}
\hline This work & \multicolumn{3}{c}{} & \\
\hline Variable & slope & constant & n. of objects & $R^{2 a}$ \\
\hline $\log \mathrm{H} \alpha+[\mathrm{NII}] \mathrm{EW}^{b}$ & $0.51 \pm 0.26$ & $19.76 \pm 0.43$ & 11 & 0.29 \\
$12+\log (\mathrm{O} / \mathrm{H})$ & $-1.01 \pm 0.14$ & $29.28 \pm 0.20$ & 12 & 0.83 \\
$\log L_{H}^{c}$ & $-0.38 \pm 0.06$ & $24.23 \pm 0.24$ & 14 & 0.75 \\
$M_{B}$ & $0.18 \pm 0.04$ & $23.77 \pm 0.28$ & 14 & 0.67 \\
\hline Literature & & & reference \\
\hline Variable & slope & constant & 1 \\
\hline $12+\log (\mathrm{O} / \mathrm{H})$ & -1.00 & 29.30 & 2 & \\
$12+\log (\mathrm{O} / \mathrm{H})$ & $-0.67 \pm 0.10$ & $26.43 \pm 0.86$ & 1 \\
$M_{B}$ & 0.20 & 24.44 & & \\
\hline
\end{tabular}

\footnotetext{
${ }^{a}$ Regression coefficient.

${ }^{b}$ In logarithmic scale (in $\AA$ ).

${ }^{c}$ In logarithmic scale (in solar units).

References: 1: Arimoto et al. (1996); 2: Wilson (1995).
} 

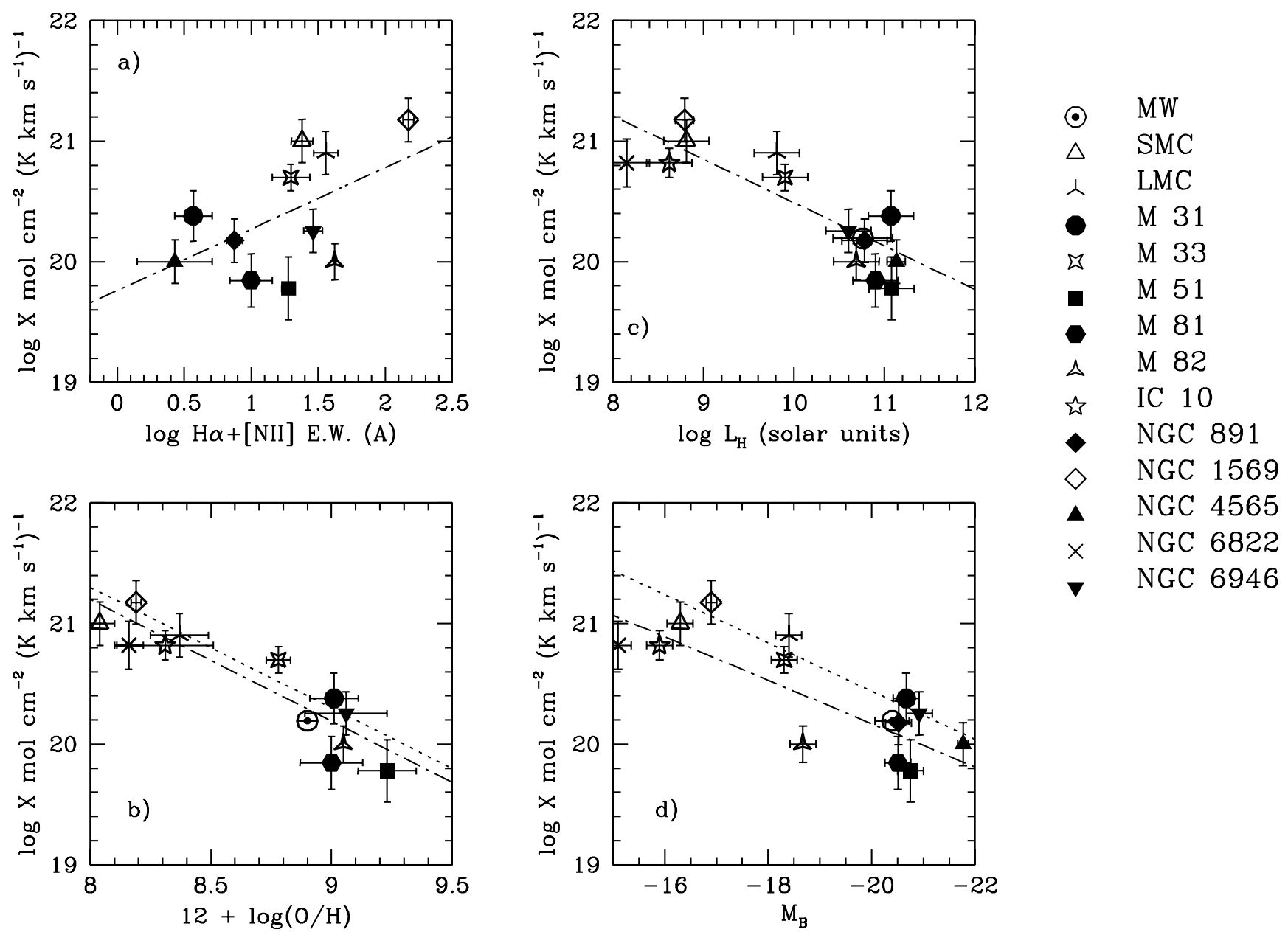

Fig. 2. The relationship for the template sample of nearby galaxies between the $X$ conversion factor from CO line intensity to $\mathrm{H}_{2}$ column density and a) the $\left.\mathrm{H} \alpha+[\mathrm{NII}] \mathrm{EW}, \mathbf{b}\right)$ the metallicity index $12+\log (\mathrm{O} / \mathrm{H})$, c) the $H$ band luminosity and d) the absolute $B$ magnitude. The dotted-dashed line is the best fit to the data; the dotted line is the best fit given by Arimoto et al. (1996).

\subsection{An alternative method}

An alternative technique for determining the molecular gas content can be pursued by assuming a metallicitydependent dust to gas ratio and determining the dust mass using far-IR or submillimetric continuum data. The dust to gas ratio is then determined in regions with no $\mathrm{CO}$ emission, hence supposed to be strongly dominated by HI. In regions with $\mathrm{CO}$ emission, the excess dust emission with respect to this ratio indicates the mass of $\mathrm{H}_{2}$. This technique has been succesfully applied to M 51, NGC 891, NGC 4565 and to some nearby irregular galaxies such as the Magellanic Clouds (Guélin et al. 1995; Guélin et al. 1993; Neininger et al. 1996; Israel 1997).

In normal galaxies such as those in our sample the dust mass is dominated by the cold dust emitting in the far-IR with a peak at $\sim 200 \mu \mathrm{m}$. The determination of the total dust mass can be achieved provided that the $100-1000 \mu \mathrm{m}$ far-IR flux and the cold dust temperature are known. Recent observations aimed at determining the spectral energy distribution in the far-IR of normal, quiescent galaxies indicate that their SED can be fitted by a modified Planck law $\nu^{\beta} B_{\nu}\left(T_{\mathrm{d}}\right)$, with $\beta=2$ (Alton et al. 2000). The total dust mass can be thus determined from the relation (Devereux \& Young 1990):

$M_{\text {dust }}=C S_{\lambda} D^{2}\left(\mathrm{e}^{a / T_{\text {dust }}}-1\right) M_{\odot}$

where $C$ is a quantity which depends on the grain opacity, $S_{\lambda}$ is the far-IR flux at a given wavelength (in Jy), $D$ is the distance of the galaxy (in Mpc), $T_{\text {dust }}$ is the dust temperature, and $a$ is a quantity which depends on $\lambda$. The majority of our sample has only IRAS data at 60 and $100 \mu \mathrm{m}$. Given the strong contamination of the emission at $60 \mu \mathrm{m}$ by very small grains, the 60 to $100 \mu \mathrm{m}$ ratio cannot be used to measure $T_{\text {dust }}$ (Contursi et al. 2001). The ISOPHOT data at 100 and $200 \mu \mathrm{m}$ give a better measure of the dust temperature. $T_{\text {dust }}$ determined for the ISOPHOT sample, as well as for 6 quiescent galaxies observed with ISOPHOT by Alton et al. (1998) consistently with Contursi et al. (2001), seems to be independent of the UV radiation field as traced by the $\mathrm{H} \alpha+[\mathrm{NII}] \mathrm{EW}$, of the metallicity or of the total luminosity. The average value is $T_{\text {dust }}=20.8 \pm 3.2 \mathrm{~K}$, which we will assume also for galaxies without ISOPHOT measurements. 


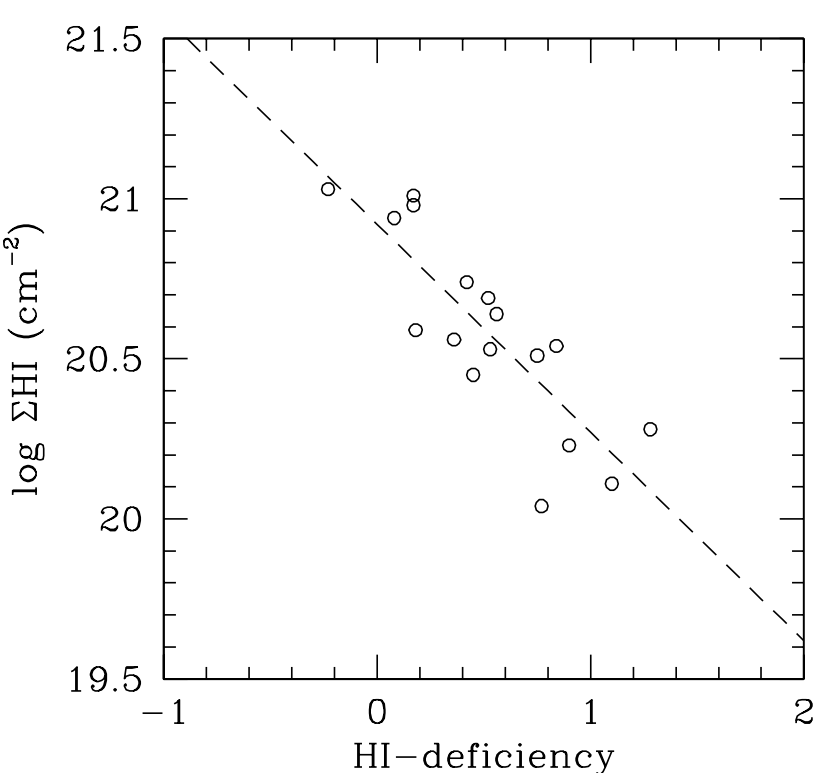

Fig. 3. The relationship between the HI surface density and the HI-deficiency parameter for the galaxies in common with Cayatte et al. (1994). The dashed line gives the best fit to the data.

We then estimate the dust mass of the sample galaxies using Eq. (4) with $C=1.27 M_{\odot} \mathrm{Jy}^{-1} \mathrm{Mpc}^{-2}$, consistent with Contursi et al. (2001), and $a=144 \mathrm{~K}$ for $S_{\lambda}=S_{100 \mu \mathrm{m}}$ (Devereux \& Young 1990).

The determination of the dust to gas ratio in a way consistent with that obtained in the solar neighbourhood, requires the estimate of the gas and dust surface densities, thus of the spatial distribution of dust and gas over the discs. Unfortunately only integrated $\mathrm{HI}$ and dust masses are available for our spatially unresolved galaxies. It is however reasonable to assume that the cold dust is as extended as the optical disc (Alton et al. 1998). The HI gas surface density is available only for a few galaxies in our sample from VLA observations (Cayatte et al. 1994). For these objects we observe a good relationship between the HI surface density $\Sigma H I$ and the HI-deficiency parameter (HI - def), (defined as in Sect. 5.1) (Fig. 3):

$\log \Sigma \mathrm{HI}=20.92( \pm 0.17)-0.65( \pm 0.11) \times(\mathrm{HI}-$ def $) \mathrm{cm}^{-2}(5)$

which can be used to predict $\Sigma H I$ for most of the galaxies of our sample with single dish HI observations.

The gas to dust ratio should depend on metallicity since the dust content is expected to be proportional to the metal content. A gas to dust vs. metallicity relation can be calibrated using the data available for the MW (Sodroski et al. 1994), the LMC (4 times solar; Koornneef 1982) and the SMC (10 times solar; Bouchet et al. 1985). This gives the relation:

$$
\begin{aligned}
\log (\text { gas } / \text { dust })= & 10.207( \pm 0.015)-1.146( \pm 0.024) \\
& \times(12+\log (\mathrm{O} / \mathrm{H}))+\log (\text { gas } / \text { dust }) \odot
\end{aligned}
$$

where the gas to dust ratio is given relative to the solar neighborhood, estimated by Sodroski et al. (1994) at

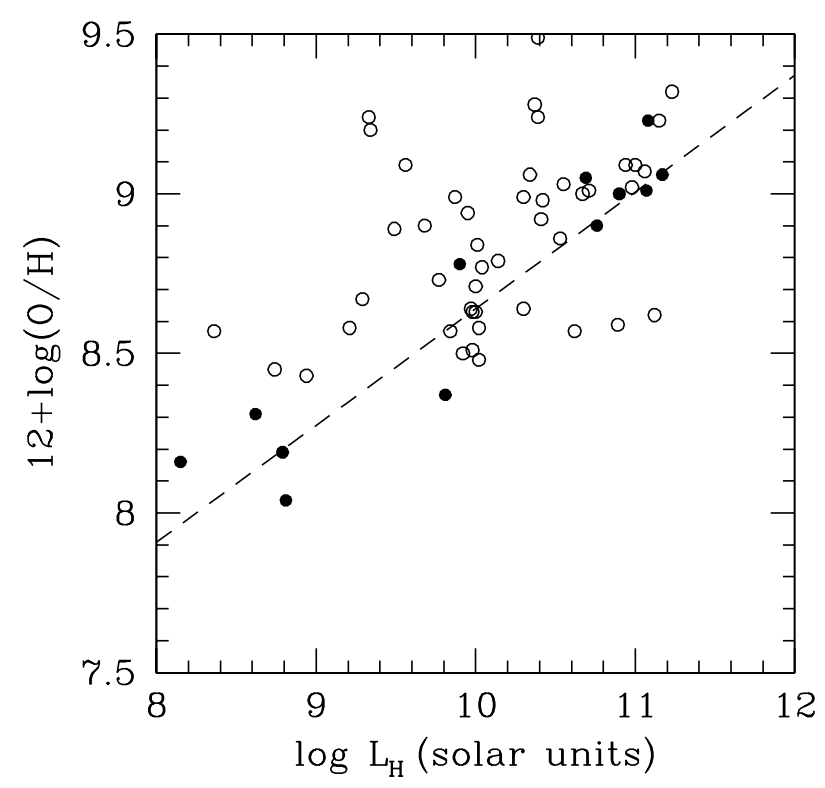

Fig. 4. The relationship between the metallicity $(12+$ $\log (\mathrm{O} / \mathrm{H}))$ and the $H$ band luminosity. Filled dots are for the nearby galaxies, empty dots for the remaining objects with metallicity measurements. The dashed line gives the best fit to the data for the nearby sample.

$(\text { gas } / \text { dust })_{\odot}=160$. The metallicity can be predicted using the metallicity vs. $H$ band luminosity relation shown in Fig. 4:

$12+\log (\mathrm{O} / \mathrm{H})=4.98( \pm 0.15)+0.37( \pm 0.04) \times \log L_{H}$.

The dispersion in the nearby sample is lower than in the other galaxies probably because of a more accurate determination of the metallicity, which has been measured from spectroscopic observations of single HII regions, while for the remaining galaxies it has been determined from long slit spectra integrated over their disc (drifting technique, Gavazzi et al. in preparation). The adopted fit is however consistent with the result of Gavazzi et al., based on a large sample of galaxies with spectroscopic measurements.

Using Eqs. (6) and (7) we can predict the gas to dust ratio for a galaxy of any $H$ luminosity. The molecular gas mass comes directly if we assume that the $\mathrm{H}_{2}$ is homogeneously distributed over the optical disc and $X$ is given by $X=M\left(\mathrm{H}_{2}\right)_{\text {dust }} / I(\mathrm{CO})$. The assumption of a homogeneous, flat distribution for the molecular hydrogen component over the disc of galaxies, which is in contradiction with the observational evidence that the $\mathrm{CO}$ emission is generally centrally peaked (see Sect. 4.1), might introduce a systematic error in the determination of $X$. We remark however that the expected $\mathrm{H}_{2}$ distribution is flatter than that of the $\mathrm{CO}$ emitting gas because of the observed decrease of the metallicity in the outer parts of galaxy discs.

The values of $X$ obtained for the sample galaxies are compared with those of the template galaxies in Fig. 5 (small symbols). In spite of the larger scatter, it is encouraging to see that the new values of $X$, at any given luminosity, metallicity and UV radiation field, are in rough agreement with those obtained for the template. The large 

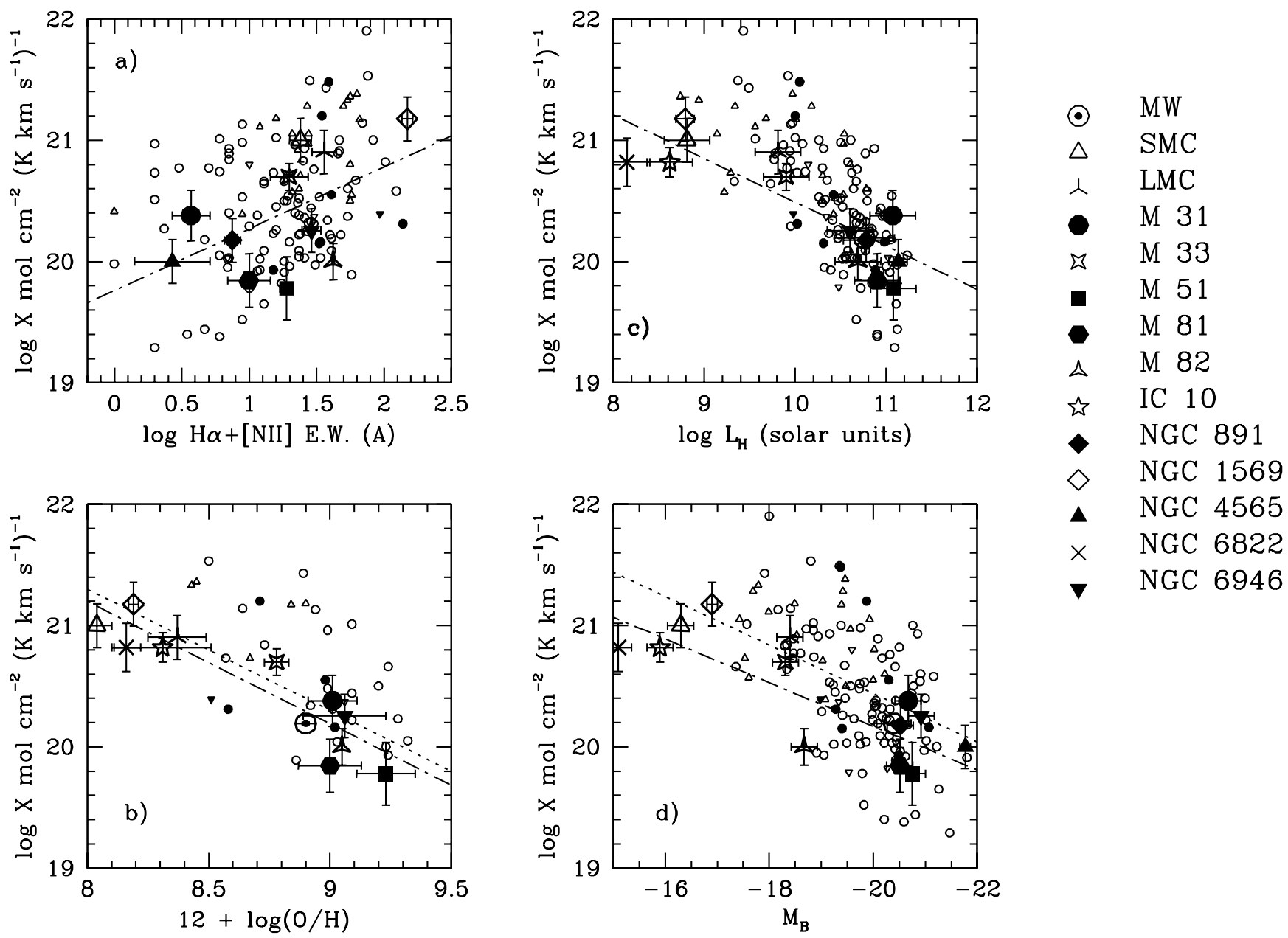

Fig. 5. Same as Fig. 2 but including values of $X$ determined using the alternative method described in Sect. 6.2 for the unperturbed sample (small symbols). Small open dots are for galaxies detected at $100 \mu \mathrm{m}$ and CO, small open triangles for galaxies undetected at $100 \mu \mathrm{m}(\nabla)$ or $\mathrm{CO}(\triangle)$. Filled symbols are for the ISOPHOT sample.

uncertainty and systematic effects are not unexpected given the number of assumptions underlying the method. From Fig. 5 we conclude that the luminosity-dependent $X$ conversion factor given in Table 5 is appropriate for estimating the molecular gas content of late-type galaxies from ${ }^{12} \mathrm{CO}(1-0)$ line intensity measurements. From now on, the molecular gas content of the 266 sample galaxies, $M\left(\mathrm{H}_{2}\right)$, is estimated using the $H$ band luminositydependent $X$ value given in Table 5 .

\section{Discussion}

\subsection{The molecular gas content of late-type galaxies}

The molecular gas content of our sample galaxies, normalized to the total mass of galaxies, is plotted in Figs. 6a and $\mathrm{b}$ as a function of the $H$ luminosity and the morphological type. To avoid any systematic environmental effect, only unperturbed galaxies with an HI-deficiency $\leq 0.3$ (defined as in Sect. 5.1) are considered.

Figure 6 shows a strong anticorrelation between the normalized molecular gas mass and the total mass of galaxies, as traced by the $H$ luminosity. The relationship with the morphological type is significantely weaker, even though it seems that early spirals have a lower normalized amount of molecular gas than late-type ones. Figures $6 \mathrm{c}$ and $\mathrm{d}$ show that the molecular to atomic hydrogen ratio is roughly constant in galaxies spanning a large range in luminosity and morphological type. The average value is $M\left(\mathrm{H}_{2}\right) / M(\mathrm{HI})=0.14$ (with upper limits treated as detections).

The weak trends of the molecular gas content (per unit mass) with the morphological type and with the $H$ luminosity as well as the constant molecular to atomic gas fraction observed by Boselli et al. (1997b) on a small, optically selected sample of Sa-Sc galaxies in the Coma supercluster is confirmed here with higher statistical significance and extended to lower luminosities, including Im and BCDs. The decrease of the molecular hydrogen to dynamical mass ratio or to the atomic hydrogen ratio claimed by Casoli et al. (1998), Sage (1993), Young \& Knezek (1989) and Kenney \& Young (1988b) for Scd-Sm-Im galaxies is probably due to a systematic underestimate of the total $M\left(\mathrm{H}_{2}\right)$ in low mass galaxies when derived assuming a constant $X$ conversion factor. 

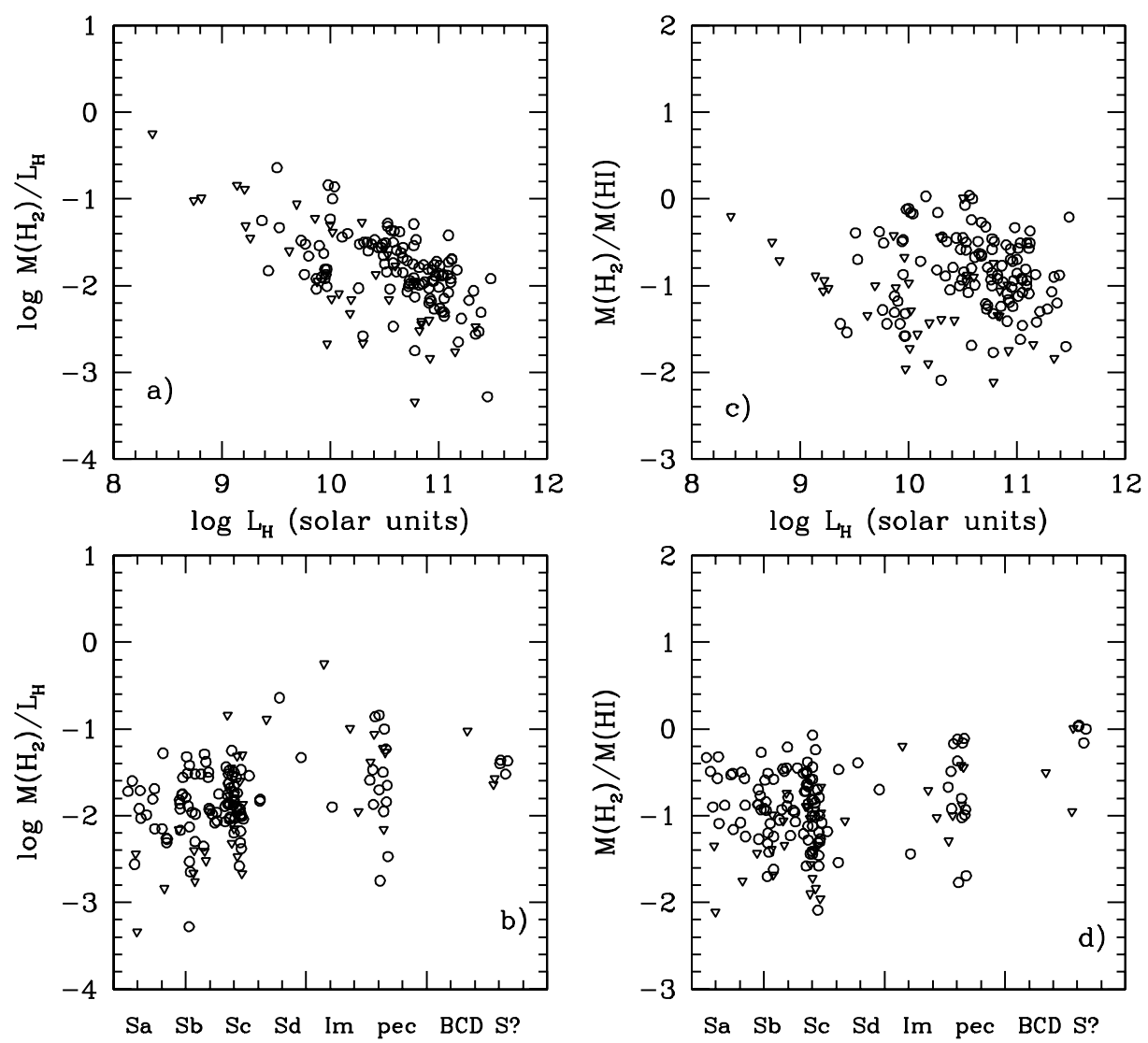

Fig. 6. The relationship between the normalised molecular gas mass of unperturbed galaxies $(\mathrm{HI}-$ deficiency $\leq 0.3)$ and a) the $H$ band luminosity, b) the morphological type; the relationship between the $\mathrm{H}_{2}$ to $\mathrm{HI}$ gas ratio and c) the $H$ band luminosity, d) the morphological type. All quantities are in solar units. Open triangles are upper limits to the molecular gas mass. To avoid overplotting, a random number between -0.4 and 0.4 was added to each half Hubble class bin taken as unity.

\subsection{The effect of the environment on the molecular gas}

The available data can be used to analyse the effects of environment on the molecular gas content of normal galaxies. Following Boselli et al. (1997b) an $\mathrm{H}_{2}$ deficiency parameter can be defined once the relationship between the molecular gas content and the $H$ luminosity is calibrated on the unperturbed sample:

$\log M\left(\mathrm{H}_{2}\right)=3.28( \pm 0.39)+0.51( \pm 0.05) \log L_{H}$

where $M\left(\mathrm{H}_{2}\right)$ and $L_{H}$ are expressed in solar units. We can thus define the $\mathrm{H}_{2}$ deficiency parameter as:

$\mathrm{H}_{2}-$ deficiency $=\log M\left(\mathrm{H}_{2}\right)_{\mathrm{e}}-\log M\left(\mathrm{H}_{2}\right)_{\mathrm{o}}$

where $M\left(\mathrm{H}_{2}\right)_{\mathrm{e}}$ is the expected molecular gas mass of a galaxy of a given $H$ luminosity as determined from Eq. (8) and $M\left(\mathrm{H}_{2}\right)_{\mathrm{o}}$ is the observed molecular gas mass. The $\mathrm{H}_{2}$ deficiency parameter determined for the whole sample is plotted in Fig. 7 versus the HI-deficiency parameter.

The present work confirms the lack of molecular-gas deficient galaxies in clusters such as Coma (Boselli et al. 1997b; Casoli et al. 1991) or Virgo (Kenney \& Young 1989; Boselli 1994), extending previous results to lower luminosities. This analysis suggests that the low luminosity,

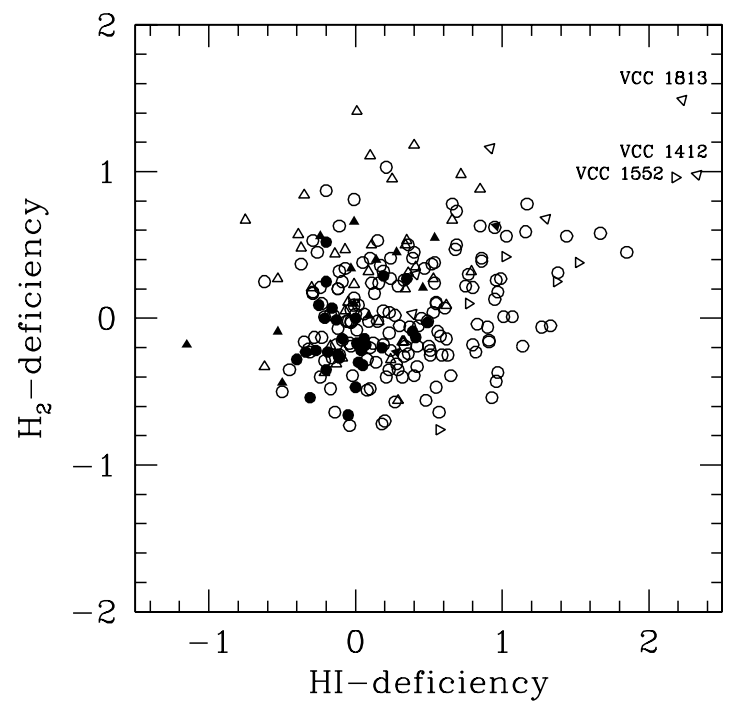

Fig. 7. The relationship between the $\mathrm{H}_{2}$ and the $\mathrm{HI}$-deficiency parameter; filled dots are for the isolated galaxies sample. Triangles indicate lower limits to the $\mathrm{H}_{2}(\triangle)$, HI $(\triangleright)$ or both gas deficiency.

CO deficient spiral galaxies observed in Virgo by Kenney \& Young (1988b) are not necessarily deficient in molecular hydrogen. 


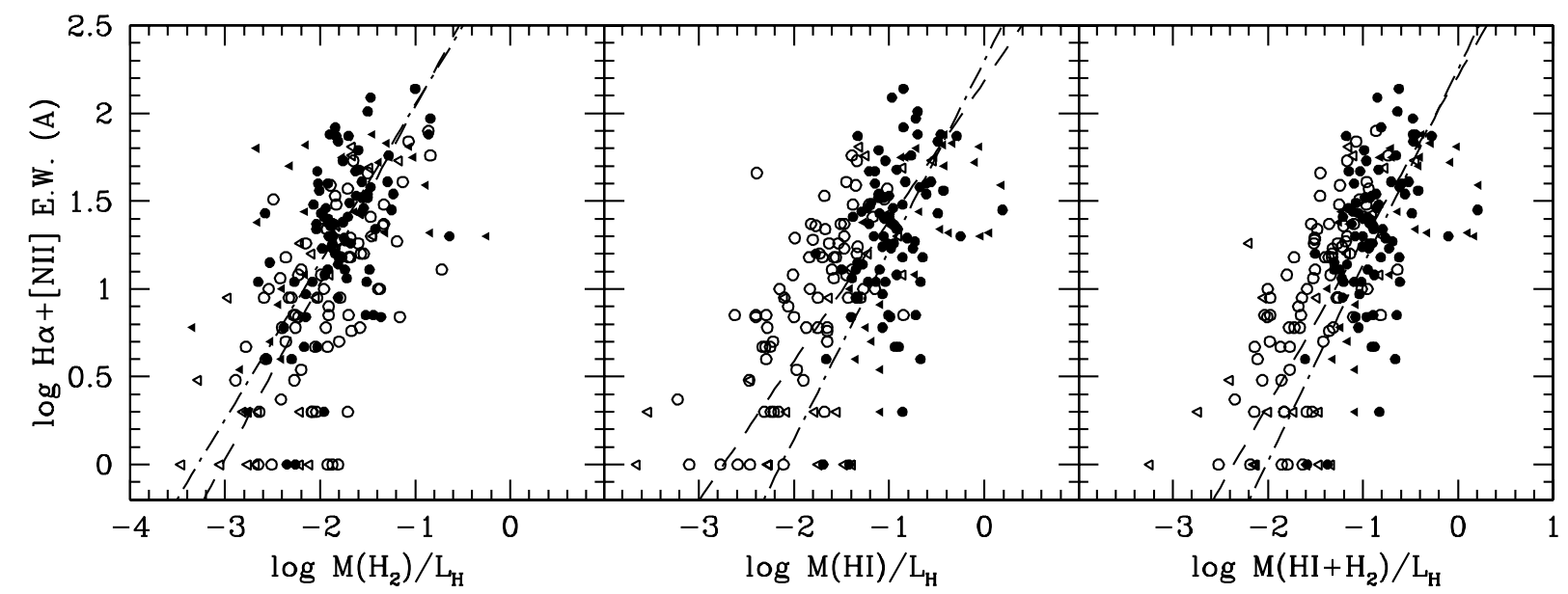

Fig. 8. The relationship between the normalized star formation index $\mathrm{H} \alpha+[\mathrm{NII}] \mathrm{EW}$ (see text) and a) the molecular, b) the atomic and c) the total gas mass normalized to the $H$ luminosity (in solar units). Filled symbols are for the unperturbed sample $(\mathrm{HI}-$ deficiency $\leq 0.3)$. Triangles indicate upper limits to the molecular hydrogen mass. The dashed line is the best fit to the whole sample, the dashed-dotted line the best fit to the unperturbed sample.

Table 6. The relationships between the $\log \mathrm{H} \alpha+[\mathrm{NII}] \mathrm{EW}$ and the atomic, molecular and total gas phases for the whole sample and for the unperturbed sample (bisector fit, with upper limits treated as detections).

\begin{tabular}{|c|c|c|c|}
\hline \multicolumn{4}{|l|}{ Whole sample } \\
\hline Variable & slope & constant & scatter \\
\hline $\log M(\mathrm{HI}) / L_{H}$ & 0.797 & 2.184 & 0.603 \\
\hline $\log M\left(\mathrm{H}_{2}\right) / L_{H}$ & 1.005 & 3.042 & 0.723 \\
\hline $\log M\left(\mathrm{HI}+\mathrm{H}_{2}\right) / L_{H}$ & 0.938 & 2.214 & 0.554 \\
\hline \multicolumn{4}{|c|}{ Unperturbed sample $(\mathrm{HI}-\operatorname{def} \leq 0.3)$} \\
\hline Variable & slope & constant & scatter \\
\hline $\log M(\mathrm{HI}) / L_{H}$ & 1.078 & 2.300 & 0.902 \\
\hline $\log M\left(\mathrm{H}_{2}\right) / L_{H}$ & 0.896 & 2.944 & 0.741 \\
\hline $\log M\left(\mathrm{HI}+\mathrm{H}_{2}\right) / L_{H}$ & 1.116 & 2.251 & 0.792 \\
\hline
\end{tabular}

\subsection{The relationship between the molecular gas content and star formation}

The atomic gas has to condense into molecular clouds to form new stars. A strong relationship between any tracer of star formation and the molecular gas content of latetype galaxies is thus expected.

The relationship between the normalized star formation index $\mathrm{H} \alpha+[\mathrm{NII}] \mathrm{EW}^{2}$ and the molecular gas content (per unit mass) observed in bright galaxies by Boselli et al. (1995b, 1997b) extends to low luminosity galaxies $\left(8 \leq \log L_{H} \leq 12 L_{H \odot}\right)$ (see Fig. 8a) once their molecular gas mass is estimated using a luminosity-dependent $X$ conversion factor. The best fits to the data along with the scatter from the linear fit are given in Table 6 for the whole sample and for the unperturbed sample $(\mathrm{HI}-\operatorname{def} \leq 0.3)$. This observational evidence confirms that the lack of a

${ }^{2}$ The $\mathrm{H} \alpha+[\mathrm{NII}] \mathrm{EW}$ is a normalized entity since it is defined as the $\mathrm{H} \alpha+[\mathrm{NII}]$ flux normalized to the underlying red continuum. strong relationship between any star formation tracer and the molecular gas mass when determined assuming a constant value of $X$ is due to a systematic underestimate of the total molecular gas mass of low luminosity galaxies. Figure 8 shows that the relationship between the star formation activity and the total gas $\left(\mathrm{HI}+\mathrm{H}_{2}\right)$ content of galaxies is stronger and less dispersed than for the individual gas components. This relationship is shared by normal and gas deficient galaxies, even though unperturbed galaxies have on average higher values of $\mathrm{H} \alpha+[\mathrm{NII}] \mathrm{EW}$.

\subsection{The star formation efficiency}

The efficiency in transforming gas into stars can be estimated using the star formation rate $(S F R)$ according to Boselli et al. (2001), i.e. using $\mathrm{H} \alpha+[\mathrm{NII}]$ fluxes corrected for the contribution of the [NII] line, for extinction, and transformed into $S F R$ (in solar masses per year) assuming a IMF of slope $\alpha=-2.5$ in the mass range $M_{\mathrm{up}}=80 M_{\odot}$ and $M_{\text {low }}=0.1 M_{\odot}$.

Figure 9 shows the relationship between the present SFE, defined as:

$$
S F E=S F R / M\left(\mathrm{H}_{2}\right)\left(\mathrm{yr}^{-1}\right)
$$

and the $H$ luminosity or the morphological type for the sample galaxies. If we were to consider the $S F E$ on cosmological timescales, the whole gas reservoir $\left(\mathrm{HI}+\mathrm{H}_{2}\right)$ would have to be taken into account, as done in Boselli et al. (2001). Figure 9 shows that the present $S F E$ is similar for galaxies of different morphological type or luminosity. This result does not change if the $S F E$ is determined using the total gas mass (Boselli et al. 2001; Boissier et al. 2001), as expected being the $\mathrm{HI}$ to $\mathrm{H}_{2}$ ratio constant (Fig. 6). Furthermore we do not see significant systematic differences in the $S F E$, as determined using $\mathrm{H}_{2}$ gas masses (Eq. (10)), for the HI-deficient objects. 

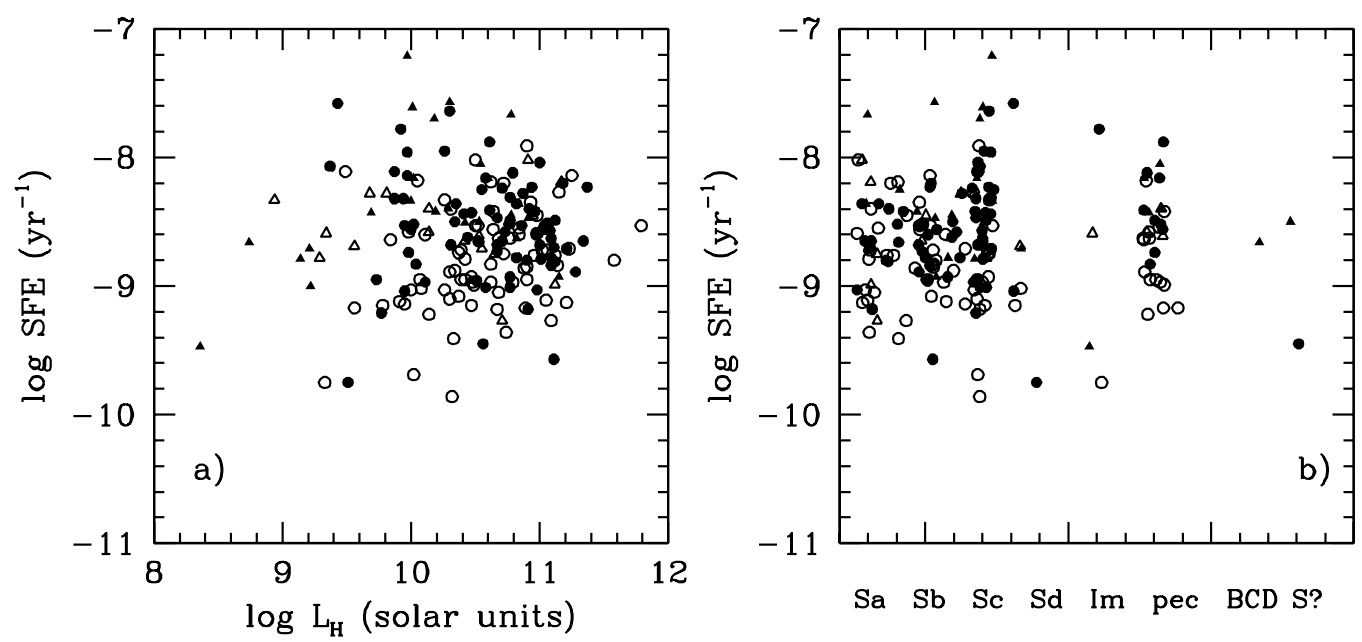

Fig. 9. The relationship between the present star formation efficiency $S F E$ and a) the $H$ luminosity and b) the morphological type. Filled symbols are for the unperturbed sample (HI - deficiency $\leq 0.3)$. Triangles indicate lower limits to the $S F E$.

\section{Summary and conclusion}

Using spectro-photometric data available in the literature for a small sample of nearby galaxies we have analysed the relationships between the $X=N\left(\mathrm{H}_{2}\right) / I(\mathrm{CO})$ conversion factor and various parameters characterizing the physical properties of the ISM. The behaviour of the nearby galaxies, whose value of $X$ has been measured by independent techniques, is compared to that of a sample of 266 latetype galaxies with available multifrequency data. For these 266 galaxies $X$ is estimated by assuming a metallicitydependent dust to gas ratio, where the dust masses have been determined using ISOPHOT and IRAS 100-200 $\mu \mathrm{m}$ data. Both samples show an anticorrelation (correlation) between $X$ and the metallicity (or the UV radiation field), with higher values of $X$ in metal-poor, star forming galaxies. These observational results can be explained if the diffuse carbon monoxide in the outskirts of the giant molecular clouds is dissociated by UV photons at a rate higher than the molecular hydrogen which, given its higher density, is more efficiently self-shielded. The ratio between $N\left(\mathrm{H}_{2}\right)$ and $I(\mathrm{CO})$ is thus expected to increase when the $\mathrm{UV}$ radiation field increases, as in star forming regions or in regions where the extinction, therefore the dust content and the metallicity, are low.

Given the strong relationship between metallicity, star formation activity and luminosity, the sample of nearby galaxies is used to calibrate a luminosity-dependent $X$ conversion factor. Using this new calibration, we reanalyse the molecular gas properties of galaxies spanning a large range in morphological type and luminosity. Lowmass, dwarf galaxies have higher molecular gas masses (per unit galaxy mass) than early-type, massive spirals. The molecular gas fraction in clouds or complexes is $\sim 15 \%$ of the total HI reservoir for all late-type galaxies.

Galaxies strongly interacting with the cluster environment have, on average, a molecular gas content comparable to isolated, unperturbed objects.
The star formation rate of late-type galaxies is strongly related to their molecular gas content. This relationship is valid for galaxies spanning a large range in luminosity.

The efficiency in transforming gas into stars is roughly constant in galaxies of different type and luminosity and belonging to different environments.

Acknowledgements. We want to thank the $12 \mathrm{~m}$ telescope operators for their invaluable help during the remote observations. We thank J. Kenney for providing us with some unpublished CO data, and C. Hoopes and N. Devereux for the $\mathrm{H} \alpha+[\mathrm{NII}] \mathrm{EW}$ of NGC 891 and M 33. We thank C. Bonfanti for providing us with some metallicity measurements, V. Buat, A. Contursi and J. M. Deharveng for interesting discussions, and S. Zibetti for providing us with fitting routines. We thank the anonymous referee for comments and suggestions which helped improving the quality of the manuscript. This publication makes use of data products from the Two Micron All Sky Survey, which is a joint project of the University of Massachusetts and the Infrared Processing and Analysis Center, funded by the National Aeronautics and Space Administration and the National Science Foundation.

\section{References}

Allen, R., \& Lequeux, J. 1993, ApJ, 410, L15

Allen, R. J., Knapen, J., Bohlin, R., \& Stecher, T. 1997, ApJ, 487, 171

Alton, P. B., Trewhella, M., \& Davies, J. I. 1998, A\&A, 335, 807

Alton, P. B., Xilouris, E. M., Bianchi, S., Davies, J. I., \& Kylafis, N. 2000, A\&A, 356, 795

Arimoto, N., Sofue, Y., \& Tsujemoto, T. 1996, PASJ, 48, 275

Bianchi, S., Davies, J., Alton, P., Gerin, M., \& Casoli, F. 2000, A\&A, 353, L13

Bicay, M., \& Giovanelli, R. 1987, ApJ, 321, 645

Binggeli, B., Sandage, A., \& Tammann, G. 1985, AJ, 90, 1681 (VCC)

Binggeli, B., Popescu, C., \& Tammann, G. 1993, A\&AS, 98, 275 
Boissier, S., Boselli, A., Prantzos, N., \& Gavazzi, G. 2001, MNRAS, 321, 733

Bolatto, A., Jackson, J., \& Ingalls, J. 1999, ApJ, 513, 275

Boselli, A. 1994, A\&A, 292, 1

Boselli, A., Casoli, F., \& Lequeux, J. 1995a, A\&AS, 110, 521

Boselli, A., Gavazzi, G., Lequeux, J., et al. 1995b, A\&A, 300, L13

Boselli, A., Tuffs, R., Gavazzi, G., Hippelein, H., \& Pierini, D. 1997a, A\&A, 121, 507

Boselli, A., Gavazzi, G., Lequeux, J., et al. 1997b, A\&A, 327, 522

Boselli, A., Gavazzi, G., Franzetti, P., Pierini, D., \& Scodeggio, M. 2000, A\&AS, 142, 73

Boselli, A., Gavazzi, G., Donas, J., \& Scodeggio, M. 2001, AJ, 121, 753

Boselli, A., \& Gavazzi, G. 2002, A\&A, submitted

Bouchet, P., Lequeux, J., Maurice, E., Prevot, L., \& PrevotBurnichon, M. 1985, A\&A, 149, 330

Casoli, F., Boissé, P., Combes, F., \& Dupraz, C. 1991, A\&A, 249, 359

Casoli, F., Sauty, S., Gerin, M., et al. 1998, A\&A, 331, 451

Cayatte, V., Kotanyi, C., Balkowski, C., \& van Gorkom, J. 1994, AJ, 107, 1003

Contursi, A., Boselli, A., Gavazzi, G., et al. 2001, A\&A, 365, 11

Devereux, N., \& Young, J. 1990, ApJ, 359, 42

Devereux, N., Price, R., Wells, L., \& Duric, N. 1994, AJ, 108, 1667

Devereux, N., Duric, N., \& Scowen, P. 1997, AJ, 113, 236

Forveille, T., Guilloteau, S., \& Lucas, R. 1990, IRAM internal report (CLASS)

Gavazzi, G., \& Boselli, A. 1996, Astrophy. Lett. Comm., 35, 1

Gavazzi, G., Boselli, A., \& Kennicutt, R. 1991, AJ, 101, 1207

Gavazzi, G., Pierini, D., Boselli A., \& Tuffs R. 1996a, A\&AS, 120,489

Gavazzi, G., Pierini, D., Baffa, C., et al. 1996b, A\&AS, 120, 521

Gavazzi, G., Catinella, B., Carrasco, L., Boselli, A., \& Contursi, A. 1998, AJ, 115, 1745

Gavazzi, G., Carrasco, L., \& Galli, R. 1999a, A\&AS, 136, 227

Gavazzi, G., Boselli, A., Scodeggio, M., Pierini, D., \& Belsole, E. 1999b, MNRAS, 304, 595

Gavazzi, G., Franzetti, P., Scodeggio, M., Boselli, A., \& Pierini, D. 2000a, A\&AS, 142, 65

Gavazzi, G., Franzetti, P., Scodeggio, M., Boselli, A., \& Pierini, D. 2000b, A\&A, 361, 863

Gavazzi, G., Boselli, A., Pedotti, P., Gallazzi, A., \& Carrasco, L. 2002, A\&A, submitted

Gezari, D., Schmitz, M., Pitts, P., \& Mead, J. 1993, NASA Ref. Pub. 1294

Guélin, M., Zylka, R., Mezger, P. G., et al. 1993, A\&A, 279, L37

Guélin, M., Zylka, R., Mezger, P. G., Haslam, C. G. T., \& Kreysa, E. 1995, A\&A, 298, L29

Haynes, M., \& Giovanelli, R. 1984, AJ, 89, 758

Haynes, M., \& Giovanelli, R. 1986, ApJ, 306, 466

Helou, G., Hoffman, G., \& Salpeter, E. 1984, ApJS, 55, 433

Hoffman, L., Helou, G., Salpeter, E., Glosson, J., \& Sandage, A. 1987, ApJS, 63, 247
Hoffman, G., Lewis, B., Helou, G., Salpeter, E., \& Williams, B. 1989, ApJS, 69, 65

Hoffman, L., Salpeter, E., Farhat, B., et al. 1996, ApJS, 105, 296

Hoopes, C., Walterbos, R., \& Rand, R. 1999, ApJ, 522, 669

Hunter, D., Hawley, W., \& Gallagher, J. 1993, AJ, 106, 1797

Hunter, S., Bertsch, D., Catelli, J., et al. 1997, ApJ, 481, 205

Israel, F. 1997, A\&A, 328, 471

Kaufman, M., Wolfire, M., Hollenbach, D., \& Luhman, M. 1999, ApJ, 527, 795

Kenney, J., \& Young, J. 1988a, ApJS, 66, 261

Kenney, J., \& Young, J. 1988b, ApJ, 326, 588

Kenney, J., \& Young, J. 1989, ApJ, 344, 171

Kennicutt, R. 1989, ApJ, 344, 685

Kennicutt, R. 1992, ApJ, 388, 310

Kennicutt, R. 1998, ARA\&A, 36, 189

Kennicutt, R., \& Kent, S. 1983, AJ, 88, 1094

Kennicutt, R., Bothun, G., \& Schommer, R. 1984, AJ, 89, 1279

Kennicutt, R., Tamblyn, P., \& Congdon, C. 1994, ApJ, 435, 22

Kennicutt, R., Bresolin, F., Bomans, D., Bothun, G., \& Thompson, I. 1995, AJ, 109, 594

Kobulnicky, H., \& Skillman, E. 1997, ApJ, 489, 636

Koornneef, J. 1982, A\&A, 107, 247

Lequeux, J., Le Bourlot, J., Pineau des Forêts, G., et al. 1994, A\&A, 292, 371

Magri, C. 1994, AJ, 108, 896

Moss, C., Whittle, M., \& Pesce, J. 1998, MNRAS, 300, 205

Neininger, N., Guélin, M., Garcia-Burillo, S., Zylka, R., \& Wielebinski, R. 1996, A\&A, 310, 725

Polk, K., Knapp, G., Stark, A., \& Wilson, R. 1988, ApJ, 332, 432

Rand, R., Kulkarni, S., \& Hester, J. 1992, ApJ, 396, 97

Rubio, M. 1999, IAUS, 190, 67

Rubio, M., Lequeux, J., Boulanger, F., et al. 1993, A\&A, 271, 1

Sage, L. 1993, A\&A, 272, 123

Schmidt, M. 1959, ApJ, 129, 243

Schneider, S., Thuan, T., Magri, C., \& Wadiak, J. 1990, ApJS, 72,245

Scodeggio, M., \& Gavazzi, G. 1993, ApJ, 409, 110

Sodroski, T., Bennett, C., Boggess, N., et al. 1994, ApJ, 428, 638

Strong, A., Bloemen, J., Dame, T., et al. 1988, A\&A, 207, 1

Taylor, C., \& Wilson, C. 1998, ApJ, 494, 581

Taylor, C., Hüttemeister, S., Klein, U., \& Greve, A. 1999, A\&A, 349, 424

Thuan, T., \& Sauvage, M. 1992, A\&AS, 92, 749

van den Bergh, S. 1999, A\&ARv, 9, 273

Walterbos, R., \& Kennicutt, R. 1987, A\&AS, 69, 331

Wild, W., Harris, A., Eckart, A., et al. 1992, A\&A, 265, 447

Wilson, C. 1995, ApJ, 448, L97

Young, J., \& Knezek, P. 1989, ApJ, 345, L55

Young, J., \& Scoville, N. 1991, ARA\&A, 29, 581

Young, J., Xie, S., Tacconi, L., et al. 1995, ApJS, 98, 219

Zaritsky, D., Kennicutt, R., \& Huchra, J. 1994, ApJ, 420, 87

Zwicky, F., Herzog, E., Karpowicz, M., Kowal, C., \& Wild, P. 1961-1968, Catalogue of Galaxies and of Cluster of Galaxies (Pasadena, California Institute of Technology; CGCG) 